Hydroécol. Appl. (1996) Tome 8 Vol. 1-2, pp. 1-33

\title{
Impact de l'élévation artificielle de température induite par le fonctionnement du Centre Nucléaire de Production Electrique du Bugey (fleuve Rhône) sur les communautés de poissons
}

\author{
Impact of thermal loading induced by the Bugey nuclear \\ power plant (upper Rhône river, France) on fish catches \\ and on fish population structure
}

\author{
V. Ginot ${ }^{(1)}$, Y. Souchon ${ }^{(2)}$ et P. Roger ${ }^{(2)}$ \\ (1) INRA, station d'hydrobiologie lacustre, BP 511 - 74203 Thonon-les-Bains, E.mail: \\ ginot@thonon.inra.fr. \\ (2) Cemagref - Groupement de Lyon, Division Biologie des Ecosystèmes Aquatiques, Labora- \\ toire d'Hydroécologie Quantitative. 3bis quai Chauveau, CP 220 - 69336 Lyon Cedex 09. \\ E.mail: yves.souchon@ cemagref.fr.
}

Résumé. - Nous présentons l'effet du réchauffement du Centre Nucléaire de Production Electrique (CNPE) du Bugey (Rhône, France) sur les communautés rivulaires de poissons. Les données ont été obtenues par pêche électrique en continu des rives de 9 stations, 4 fois par an, de 1979 à 1994, soit 519 pêches et 46356 poissons. Les poissons quittent progressivement les bordures dès que la température dépasse 15 degrés. Un seuil est atteint à $25^{\circ} \mathrm{C}$ où les animaux les plus gros désertent la zone. La chute des effectifs est alors de plus en plus rapide et un deuxième seuil apparaît à $29^{\circ} \mathrm{C}$ avec l'effondrement du nombre d'espèces. La composition des pêches dépend de la température uniquement pour les adultes, avec trois grands groupes, moins de $6^{\circ} \mathrm{C}, 7$ à $24^{\circ} \mathrm{C}$, et plus de $25^{\circ} \mathrm{C}$. Ces trois groupes restent dans l'ordre biotypologique O-IV de Verneaux (1977) typique du haut Rhône. Des modifications importantes de structures liées au débit et à la saison sont observées, et contribuent à masquer l'effet thermique dans le groupe central. Ces trois facteurs expliquent $30 \%$ de la variabilité des données. L'attrait hivernal pour les veines réchauffées apparaît quantitativement nul, et ne concerne structurellement que la perche que ce contraste semble attirer. Les captures de juvéniles sont extrêmement variables et leur composition ne peut pas être reliée à la température. Le site du Bugey paraît bien supporter la charge thermique du CNPE, peut-être en partie du fait du mauvais mélange des masses d'eau à la sortie de la centrale.

Mots clés. - Température, Réchauffement, Poisson, Peuplement, Rhône, Centrale nucléaire. 


\begin{abstract}
Thermal loading due to operation of the Bugey nuclear power plant on the Rhône river is discussed in relation to the number of fish catches and to the structure of fish populations. Data was collected by means of continuous electrofishing along the shore at 9 sites, 4 times yearly from 1979 to 1994, representing 519 samples and 46,356 fish caught. Above $15^{\circ} \mathrm{C}$, fish gradually leave the shoreline. A threshold is reached at $25^{\circ} \mathrm{C}$ when the largest individuals leave the area. The drop in numbers is increasingly rapid up to a second threshold at $29^{\circ} \mathrm{C}$, which diversity is non-existent. The composition of catches depends on temperature only for adults, with three major thermal groups appearing : below $6{ }^{\circ} \mathrm{C}$, from 7 to $24^{\circ} \mathrm{C}$, and over $25^{\circ} \mathrm{C}$. These all remain in Verneaux's 0-IV biotypological order (1977) which is typical of the upper Rhône. Secondary factors like discharge and season also have a significant impact on population structures and contribute to masking the thermal impact in the middle thermal group. These three factors explain $30 \%$ of the variance in the data. In winter, attraction for heated water appears quantitatively insignificant, except for perch which are attracted by the contrast in temperature. Catches of juveniles are extremely variable and their composition cannot be related to temperature. The Bugey site appears to stand up well to thermal loading induced by the plant, perhaps in part because mixing of the water masses at different temperatures at the outlet from the plant is not total.
\end{abstract}

Key words. - Temperature, Thermal loading, Fish assemblages, Rhône river, Nuclear power plant.

\section{INTRODUCTION}

L'analyse des impacts des aménagements de cours d'eau bénéficie rarement d'un recul temporel suffisant pour pouvoir tenir compte à la fois des fluctuations naturelles des communautés de poisson et des changements réellement induits de leur dynamique. Persat (1988), dans sa thèse sur les poissons du Haut-Rhône français, estime à huit ans la durée d'un cycle biologique significatif, alors que les suivis réglementaires des incidences des ouvrages hydroélectriques construits par la Compagnie Nationale du Rhône n'avaient été instaurés que sur 4 années. Ce défaut de suivi biologique à long terme est discuté par Magnuson (1990) qui introduit la notion de "présent invisi- ble ", mettant en garde sur les risques d'interprétations erronées de phénomènes observés trop ponctuellement. Les suivis annuels réglementaires des sites électronucléaires d'Electricité de France, conduits sans discontinuité depuis leur implantation, n'entrent plus dans cette catégorie. Ils offrent ainsi l'occasion unique d'interpréter des séries biologiques obtenues sur plus de 10 ans.

L'exemple traité concerne le Centre Nucléaire de Production Électrique (CNPE) du Bugey installé sur le Rhône en amont de Lyon, pour lequel un programme de suivi hydrobiologique a été élaboré à la fin de l'année 1978. Ce dernier comprend la physico-chimie des eaux, les invertébrés benthiques, la flore et les poissons. Différentes informations concernant 
notamment les invertébrés ont été produites par Khalanski (1988), Roger et Faessel (1989) et Roger et al. (1991). L'étude piscicole porte sur les données récoltées depuis 1979, année de mise en service effective des 3 tranches en circuit ouvert de la centrale du Bugey, jusqu'en 1994, soit sur 16 années, 519 pêches, plus de 46000 poissons ( 30226 adultes et 16130 juvéniles) et 33 espèces recensées (Tableau 1). Après une présentation du site, des conditions thermiques naturelles et induites par la centrale et du protocole des pêches, les résultats porteront d'abord sur les effets quantitatifs liés aux températures ou au contraste thermique entre secteurs témoins et réchauffés, puis sur les modifications de la composition des pêches, en lien avec la température, le débit et la saison.

\section{LE SITE DU BUGEY ET LE PROTOCOLE DES PÊCHES}

Au niveau du Bugey, le Rhône coule à 190 mètres d'altitude sur des dépôts Wurmiens et des alluvions récentes. Sa pente est de 0,35\%, sa largeur de 120 mètres, et sa profondeur moyenne de 3 mètres pour un débit moyen annuel de $445 \mathrm{~m}^{3} / \mathrm{s}$ avant la confluence de l'Ain. Le substrat est principalement constitué de gros galets au centre du lit, et de galets plus petits, de graviers et de sables en bordure. Des sédiments plus fins, limons et vases sont épisodiquement déposés et remis en suspension à l'occasion des vidanges de barrages ou des crues. Ce secteur non ralenti est moins exposé au colmatage du substrat que les retenues situées en amont. Le lit est à chenal unique relativement rectiligne sauf à l'amont de St-Vulbas où existent un bras secondaire et une île. La rive est majoritairement boisée sur une largeur de plusieurs mètres. La vitesse du courant est élevée, $1 \mathrm{~m} / \mathrm{s}$ environ. Le régime hydrologique est de type nivo-glaciaire avec un maximum estival de $600 \mathrm{~m}^{3} / \mathrm{s}$ en juillet et un minimum hivernal de $350 \mathrm{~m}^{3} / \mathrm{s}$ entre octobre et janvier. Ce régime est modifié par la gestion des nombreux ouvrages situés en amont pouvant provoquer des marnages hebdomadaires de l'ordre du mètre. L'eau reste fraîche, avec une moyenne mensuelle de $4{ }^{\circ} \mathrm{C}$ en janvier et un peu plus de $18^{\circ} \mathrm{C}$ en juillet-août. La température de surface la plus élevée enregistrée au cours des pêches dans le secteur non réchauffé est de $24^{\circ} \mathrm{C}$. La physico-chimie indique un secteur assez dégradé avec de fortes teneurs en ammoniaque et phosphate, témoins d'une pollution organique sensible (Roger et al., 1991). Ce verdict est confirmé par un peuplement d'invertébrés benthiques constitué d'espèces résistantes à la pollution, et ceci dès l'origine de l'étude. Les teneurs en oxygène restent cependant excellentes.

\subsection{Rejets thermiques du CNPE du Bugey}

Pendant la période d'étude, le Centre Nucléaire de Production Electrique du Bugey comportait 5 tranches et rejetait en moyenne $130 \mathrm{~m}^{3} / \mathrm{s}$ d'eau 
Tableau 1. - Liste des espèces recensées au cours du suivi hydrobiologique de la centrale du Bugey (fleuve Rhône, période 1979-1994). Code des espèces, seuil arbitraire de taille entre adultes et juvéniles, et nombre d'individus pêchés (effectifs normalisés à un effort de pêche de 30 minutes).

Table 1. - List of the fish species encountered during the hydrobiological monitoring of the Bugey nuclear powerplant, river Rhône, 1979-1994, with the french species code, the arbitrary length limit between adults and juveniles, and the number of catches standardized to a fishing effort of $30 \mathrm{mn}$.

\begin{tabular}{|c|c|c|c|c|c|}
\hline & & & & & \\
\hline Nom commun & Nom latin & $\begin{array}{l}\text { Code } \\
\text { espèce }\end{array}$ & $\begin{array}{l}\text { Seuil } \\
\text { A./J. } \\
(\mathrm{mm})\end{array}$ & $\begin{array}{l}\text { Nombre } \\
\text { adultes } \\
\text { pêchés }\end{array}$ & $\begin{array}{c}\text { Nombre } \\
\text { juvéniles } \\
\text { pêchés }\end{array}$ \\
\hline Chevaine & Leuciscus cephalus & $\mathrm{CHE}$ & 100 & 7602 & 4046 \\
\hline Ablette & Alburnus alburnus & $\mathrm{ABL}$ & 50 & 6662 & 1529 \\
\hline Vandoise & Leuciscus leuciscus & VAN & 100 & 5735 & 3604 \\
\hline Spirlin & Alburnoides bipunctatus & SPI & 50 & 5067 & 1236 \\
\hline Goujon & Gobio gobio & ¿ GOU & 60 & 2421 & 334 \\
\hline Barbeau fluviatile & Barbus barbus & BAF & 100 & 1986 & 1514 \\
\hline Gardon & Rutilus rutilus & GAR & 100 & 1250 & 2383 \\
\hline Hotu & Chondrostoma nasus & HOT & 100 & 973 & 1545 \\
\hline Brochet & Esox lucius & BRO & 250 & 479 & 33 \\
\hline Truite fario & Salmo trutta (fario) & TRF & 80 & 372 & 38 \\
\hline Blageon & Leuciscus souffia & . BLN & 80 & 326 & 328 \\
\hline Perche commune & Perca fluviatilis & PER & 100 & 300 & 86 \\
\hline Perche soleil & Lepomis gibbosus & PES & 60 & 128 & 38 \\
\hline Ombre & Thymallus thymallus & OBR & 100 & 85 & 4 \\
\hline Tanche & Tinca tinca & TAN & 80 & 65 & 16 \\
\hline Poisson chat & Ictalurus melas & $\mathrm{PCH}$ & 50 & 64 & 3 \\
\hline Brème bordelière & Blicca bjoerkna & BRB & 80 & 41 & 26 \\
\hline Anguille & Anguilla anguilla & ANG & 200 & 27 & 2 \\
\hline Loche & Noemacheilus barbatulus & LOF & 50 & 26 & 2 \\
\hline Bouvière & Rhodeus sericeus & $\mathrm{BOU}$ & 50 & 21 & 27 \\
\hline Carpe & Cyprinus carpio & $\mathrm{cco}$ & 80 & 20 & 1 \\
\hline Gremille & Gymnocephalus cernuus & GRE & 80 & 20 & 1 \\
\hline
\end{tabular}




\begin{tabular}{|c|c|c|c|c|c|}
\hline Nom commun & Nom latin & $\begin{array}{l}\text { Code } \\
\text { espèce }\end{array}$ & $\begin{array}{l}\text { Seuil } \\
\text { A./J. } \\
\text { (mm) }\end{array}$ & $\begin{array}{l}\text { Nombre } \\
\text { adultes } \\
\text { pêchés }\end{array}$ & $\begin{array}{c}\text { Nombre } \\
\text { juvéniles } \\
\text { péchés }\end{array}$ \\
\hline Rotengle & Scardinius erythrophtalmus & ROT & 100 & 20 & 18 \\
\hline Lote de rivière & Lota lota & LOT & 100 & 16 & 0 \\
\hline Epinoche & Gasterosteus aculeatus & EPI & 50 & 10 & 26 \\
\hline Sandre & Stizostedion lucioperca & SAN & 120 & 7 & 0 \\
\hline Black Bass & Micropterus salmoides & $B B G$ & 80 & 6 & 0 \\
\hline Vairon & Phoxinus phoxinus & VAl & 50 & 5 & 525 \\
\hline Chabot & 'Cottus gobio & $\mathrm{CHA}$ & 40 & 4 & 1 \\
\hline Toxostome & Chondrostoma toxostoma & TOX & 100 & 3 & 7 \\
\hline Brème commune & Abramis brama & BRE & 80 & 3 & 3 \\
\hline Carassin & Carassius carassius & CAS & 40 & 3 & 0 \\
\hline \multirow[t]{2}{*}{ Pseudorasbora } & Pseudorasbora parva & PSR & 40 & \multirow{2}{*}{$\begin{array}{r}0 \\
33747\end{array}$} & 1 \\
\hline & Total (effectifs normalisés) & 33 & & & 17377 \\
\hline
\end{tabular}

réchauffée d'environ $10^{\circ} \mathrm{C}$. La tranche 1 prélève $30 \mathrm{~m}^{3} / \mathrm{s}$, les tranches 2 et 3 prélèvent $46 \mathrm{~m}^{3} / \mathrm{s}$ chacune; ce débit est restitué au Rhône par un court canal de rejet, sur lequel se trouve la station 4 . Les tranches 4 et 5 sont équipées de tours de refroidissement, elles évacuent un débit total de 5 à $6 \mathrm{~m}^{3} / \mathrm{s}$ dans un rejet secondaire situé à 200 mètres à l'amont du canal de rejet principal, (fig. 1). La tranche 1 est arrêtée depuis mai 1994. D'après les profils thermiques effectués par EDF et les mesures prises par le Cemagref, il ressort que la tache thermique s'étale très vite dès la sortie du rejet sur plus de $25 \mathrm{~m}$ de large en rive droite, avec des températures maximales sur une largeur de 15 mètres. Les isothermes sont relativement verticaux (la tache thermique ne s'étale pas en surface) si bien qu'une simple mesure de surface est représentative de la colonne d'eau. Le mélange s'effectue mal, et tout se passe comme si un "Rhône chaud" coulait parallèlement à un "Rhône froid", en se diluant et en se refroidissant lentement. La veine chaude, qui se refroidit d'environ $1{ }^{\circ} \mathrm{C}$ par kilomètre, n'atteint qu'exceptionnellement la rive gauche (étiages hivernaux très sévères), et le différentiel thermique n'excède vraisemblablement plus $1^{\circ} \mathrm{C}$ en moyenne avant le mélange total à la confluence de l'Ain. 


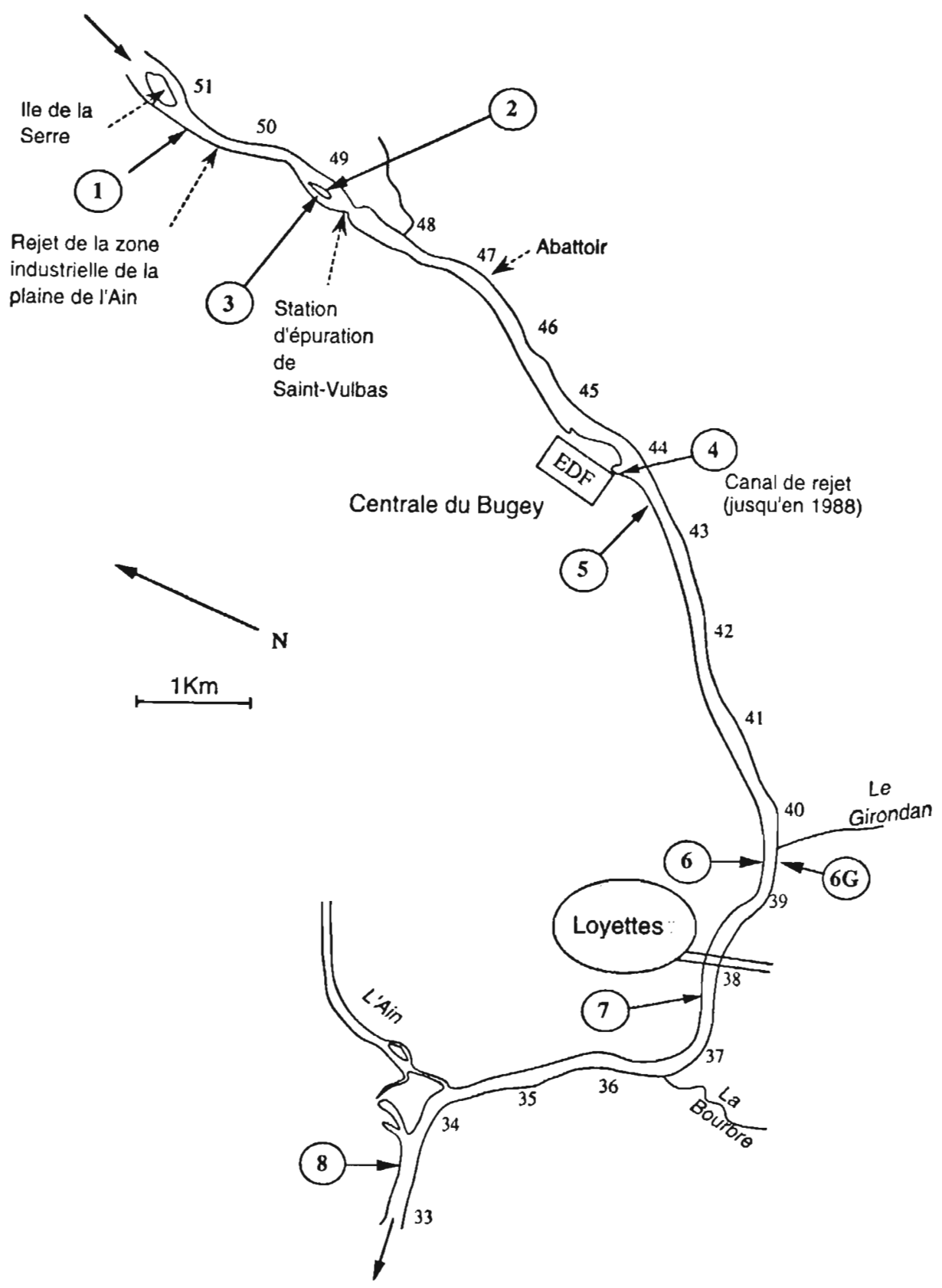

Fig. 1. - Localisation de la centrale électrique du Bugey sur le Rhône, et position des 9 stations de pêche.

Fig. 1. - Location of the Bugey power plant on river Rhône, and of the 9 sampling sites. 


\subsection{Stations d'étude}

Neuf stations d'une longueur moyenne de 500 mètres sont prospectées à chaque campagne (fig. 1). Trois stations (1-2-3) sont situées à l'amont de la centrale et servent de référence, 4 stations (4-5-6-7) s'échelonnent le long du panache thermique, une station (6G) est située en rive gauche non réchauffée juste en face de la station 6 pour compléter les stations témoins, et une dernière station (8) est située à l'aval de la confluence de l'Ain pour une référence après mélange des eaux. Le temps de pêche moyen est de trente minutes par station. Une campagne est réalisée par saison, soit 4 campagnes par an.

La station 1 présente une berge limoneuse rectiligne pratiquement sans refuges, la station 2 , dans le bras d'une île, s'envase au fil des ans et présente de ce fait des hauteurs d'eau souvent très faibles. La station 3 , à l'extérieur de l'île, présente des bancs de petits galets, des profondeurs plus importantes et quelques refuges. La station $6 \mathrm{G}$ bénéficie d'une morphologie favorable au poisson (galets, embâcles), mais, située en rive concave, elle est pénalisée aux forts débits par des vitesses et des hauteurs d'eau importantes. Les stations réchauffées ont une morphologie qui paraît globalement plus favorable aux poissons, avec des abris constitués d'embâcles ou de petits enrochements (surtout sur 5 et 7), et des vitesses et des profondeurs plus en accord avec les exigences des poissons. La station 6 , située en rive convexe, est assez en- vasée mais présente de nombreux embâcles.

D'après les températures relevées au cours de nos pêches, le rejet (station 4) affiche une augmentation moyenne de $9,7^{\circ} \mathrm{C}$ par rapport aux 3 stations amont, la station $5:+7,4^{\circ} \mathrm{C}$, la station $6:+4,5^{\circ} \mathrm{C}$, et la station 7 : $+3,1^{\circ} \mathrm{C}$ (fig. 2a). Le contraste thermique entre stations amont et stations réchauffées reste étonnamment stable au cours des quatre saisons, alors qu'on aurait pu s'attendre à ce qu'il soit plus marqué en hiver du fait du moindre débit du Rhône et de la plus forte demande en électricité (fig. 2b). La station $6 \mathrm{G}$ se comporte comme les stations amont. La station 8, à l'aval de la confluence de l'Ain, accuse une légère différence avec les stations témoins en hiver et au printemps, mais soumise à l'influence thermique de l'Ain, il est difficile d'y lire l'effet du réchauffement.

\subsection{Protocole d'échantillonnage des poissons}

Les pêches électriques sont effectuées en bateau, en continu le long des berges. Un courant triphasé est fourni par un groupe électrogène de $3 \mathrm{KVA}$, puis redressé au moyen d'un boîtier Héron II. L'anode est constituée de 2 cercles concentriques en acier inoxydable suspendus à une potence, à l'avant du bateau, et la cathode de tresses en cuivre fixées sur les flancs. Les poissons sont capturés au voisinage de l'anode dont le rayon d'action est de 2 mètres, à l'aide d'épuisettes à mailles de $5 \mathrm{~mm}$. Les pois- 


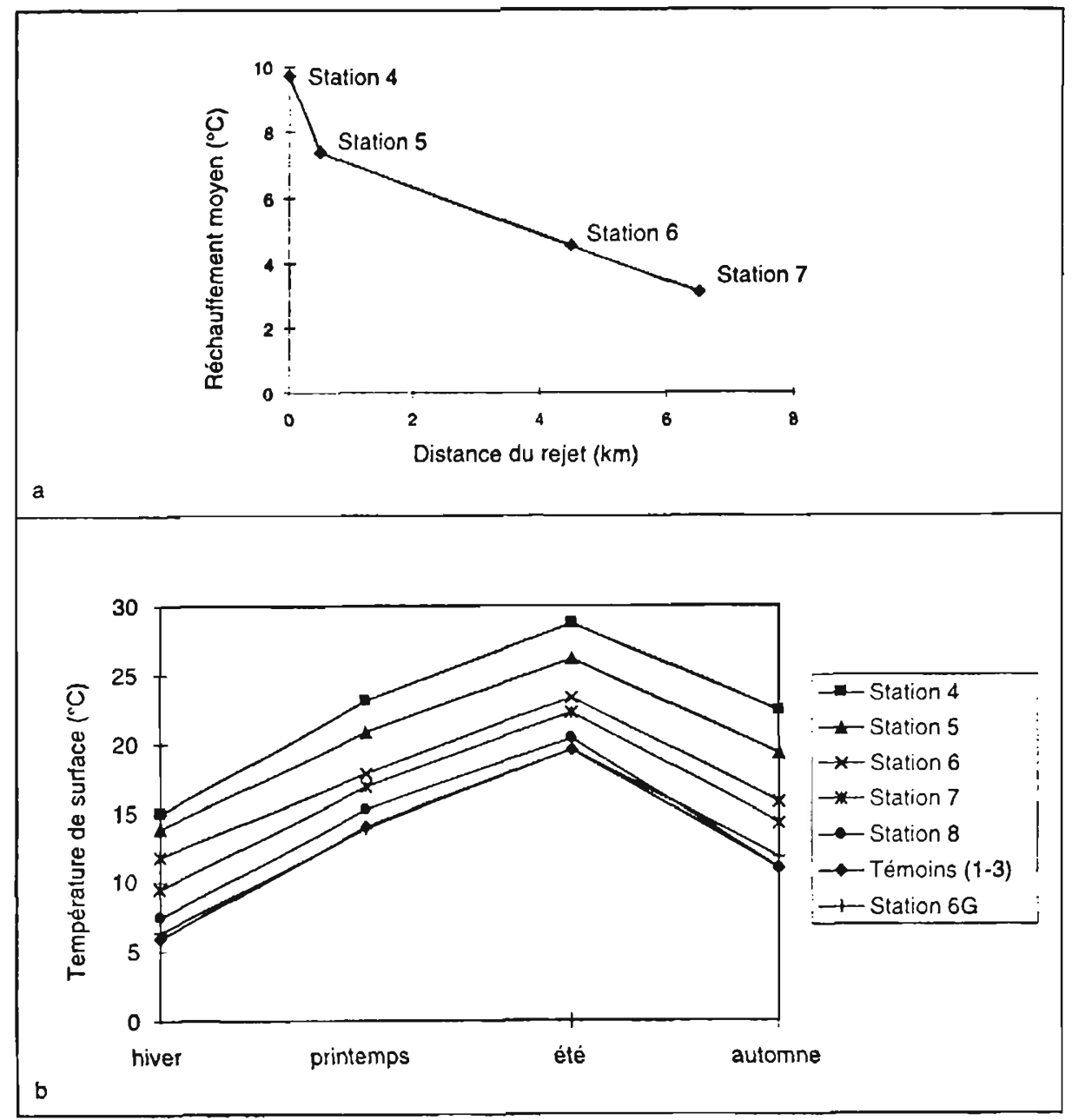

Fig. 2. - Températures de surface relevées lors des pêches sur les différentes stations. a : réchauffement moyen des stations réchauffées par rapport aux stations amont; b : température moyenne des stations en fonction des saisons.

Fig. 2. - Surface temperature of the sampling sites during fishing. a : mean increase of temperature on heated sites; $b$ : mean temperature of each site versus seasons.

sons sont identifiés à l'espèce. La taille, le poids et le cas échéant l'état pathologique des sujets sont notés. Nous regroupons de manière arbitraire sous le terme "juvénile", les individus dont la taille est inférieure à celle donnée au tableau 1 . On y trouvera des individus de l'année ou d'un an qui n'ont en principe pas encore atteint l'âge de première reproduction. Les données sont normalisées à un effort de pêche de $30 \mathrm{mn}$. 


\section{EFFET QUANTITATIF DES TEMPERATURES}

\subsection{Effet du niveau thermique}

Les biomasses pêchées en période estivale sur les stations réchauffées accusent une chute brutale entre 23 et $25^{\circ} \mathrm{C}$, avec une allure triangulaire des données, caractéristique d'un facteur limitant à contrainte croissante (fig. 3a). Cette chute est causée par le départ de gros individus car les figures $3 b$ et $3 c$ relatives aux effectifs d'adultes et de juvéniles pendant les pêches d'été, ne montrent pas de seuil à cette température, mais une décroissance linéaire (échelle logarithmique pour les effectifs) régulière avec la température. Ces deux tendances sont hautement significatives bien que l'effet négatif de la température soit moins sensible sur les juvéniles que sur les adultes du fait du moindre effectif des premiers. Si l'on élimine cet effet "effectifs" en normalisant le logarithme des effectifs par la moyenne de chaque groupe, l'hypothèse d'égalité des pentes entre adultes et juvéniles ne peut être rejetée au risque de $5 \%$. Les valeurs sont plus incertaines pour les juvéniles du fait d'effectifs moins importants que pour les adultes, et surtout d'une variabilité plus grande entre pêches ( $17 \%$ de pêches nulles contre $0,6 \%$ pour les adultes). Ce même effet identique entre adultes et juvéniles se retrouve aussi sur les stations témoins en été, mais avec une pente moindre et une incertitude accrue du fait d'une gamme thermique plus étroite et d'effectifs moins importants. La figure 4 qui reprend la moyenne de l'ensemble des pêches par groupes thermiques de $3^{\circ} \mathrm{C}$ confirme ces tendances: maintien du ratio adultes/juvéniles autour de 2/3-1/3 sur toute la gamme thermique (fig. 4c), maximum des captures pour les adultes entre 7 et $15^{\circ} \mathrm{C}$ pour les stations réchauffées (fig. 4a), maximum plus large et plus chaud (entre 9 et $19^{\circ} \mathrm{C}$ ) pour les juvéniles (fig. $4 \mathrm{~b}$ ), tendances moins marquées sur les stations témoins du fait d'effectifs moindres, surtout en ce qui concerne les juvéniles où le groupe $7-9^{\circ} \mathrm{C}$ qui semble s'individualiser reflète en fait quelques pêches exceptionnelles (ex. 1300 poissons le $13 / 3 / 90$, dont 500 petits gardons, 300 hotus et 250 chevaines). Bien qu'il existe un effet négatif de la température sur les effectifs, et probablement dès $15^{\circ} \mathrm{C}$ pour les adultes, les effectifs sont plus importants sur les stations réchauffées que sur les témoins, même si cette différence tend logiquement à s'estomper pour les températures les plus fortes (fig. 2a). Nous verrons au paragraphe suivant que cela n'est pas imputable au contraste thermique entre stations qui ferait que les poissons seraient attirés par les veines d'eau réchauffées en hiver. II semble que cela tienne aux meilleures conditions d'habitat des stations réchauffées. Elles affichent effectivement plus d'abris (embâcles, enrochements) que les stations témoins, et peuvent de ce fait abriter des effectifs plus importants tant que la contrainte thermique n'est pas trop forte. 


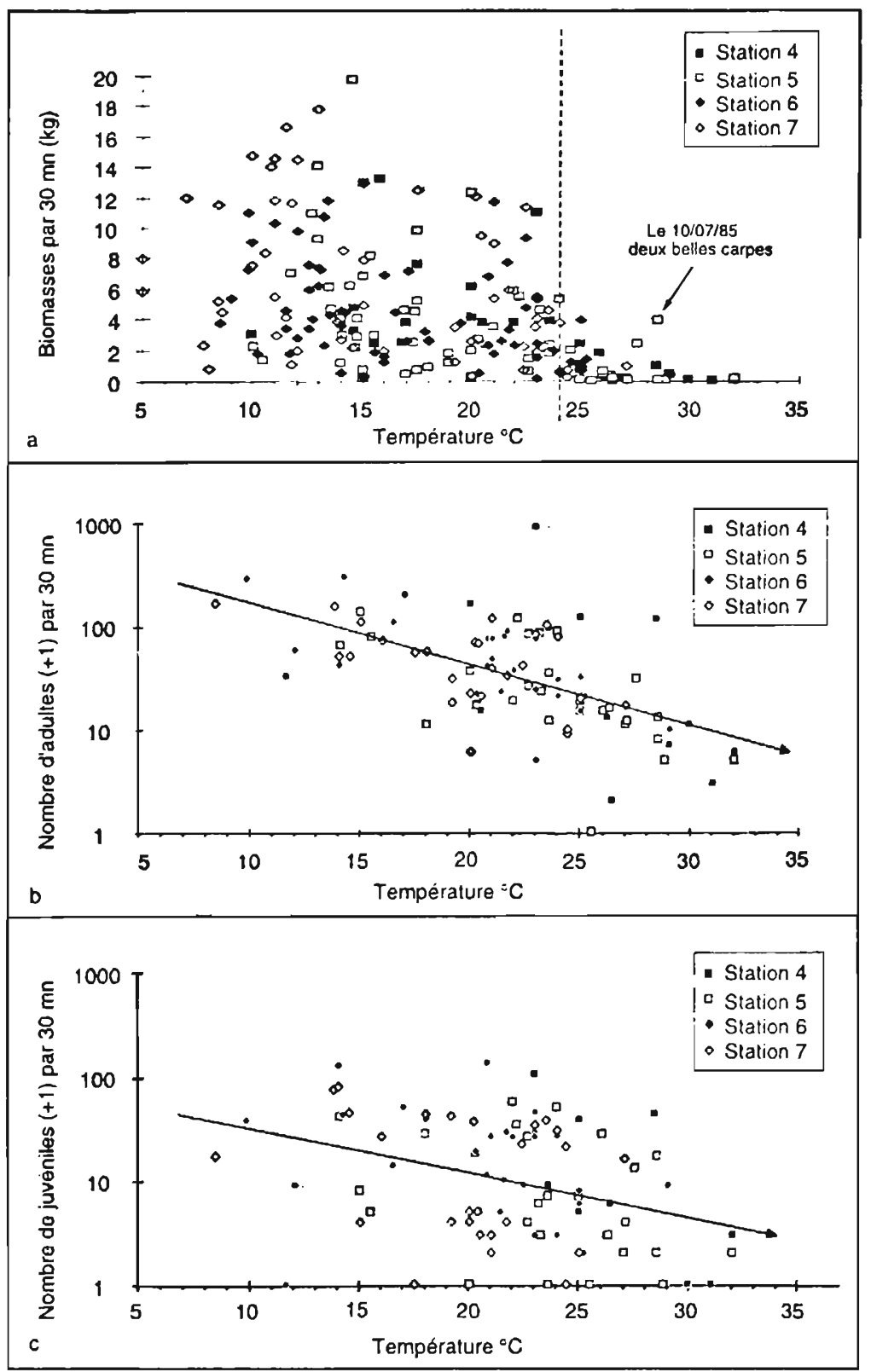

Fig. 3. - Résultats des pêches en fonction de la température, toutes espèces confondues, sur les 4 stations réchauffées. a : biomasses, toutes campagnes confondues; $b$ : effectifs d'adultes et $c$ : effectifs de juvéniles, lors des campagnes de printemps et d'été (avril à septembre). Données normées à un effort de pêche de $30 \mathrm{mn}$.

Fig. 3. - Total catches versus temperature on the 4 heated sites. a : total biomasses; $b$ : number of adultes during spring and summer fishing (april to september); $c$ : number of juveniles during the same periode. Data are standardized to a fishing effort of $30 \mathrm{mn}$. 


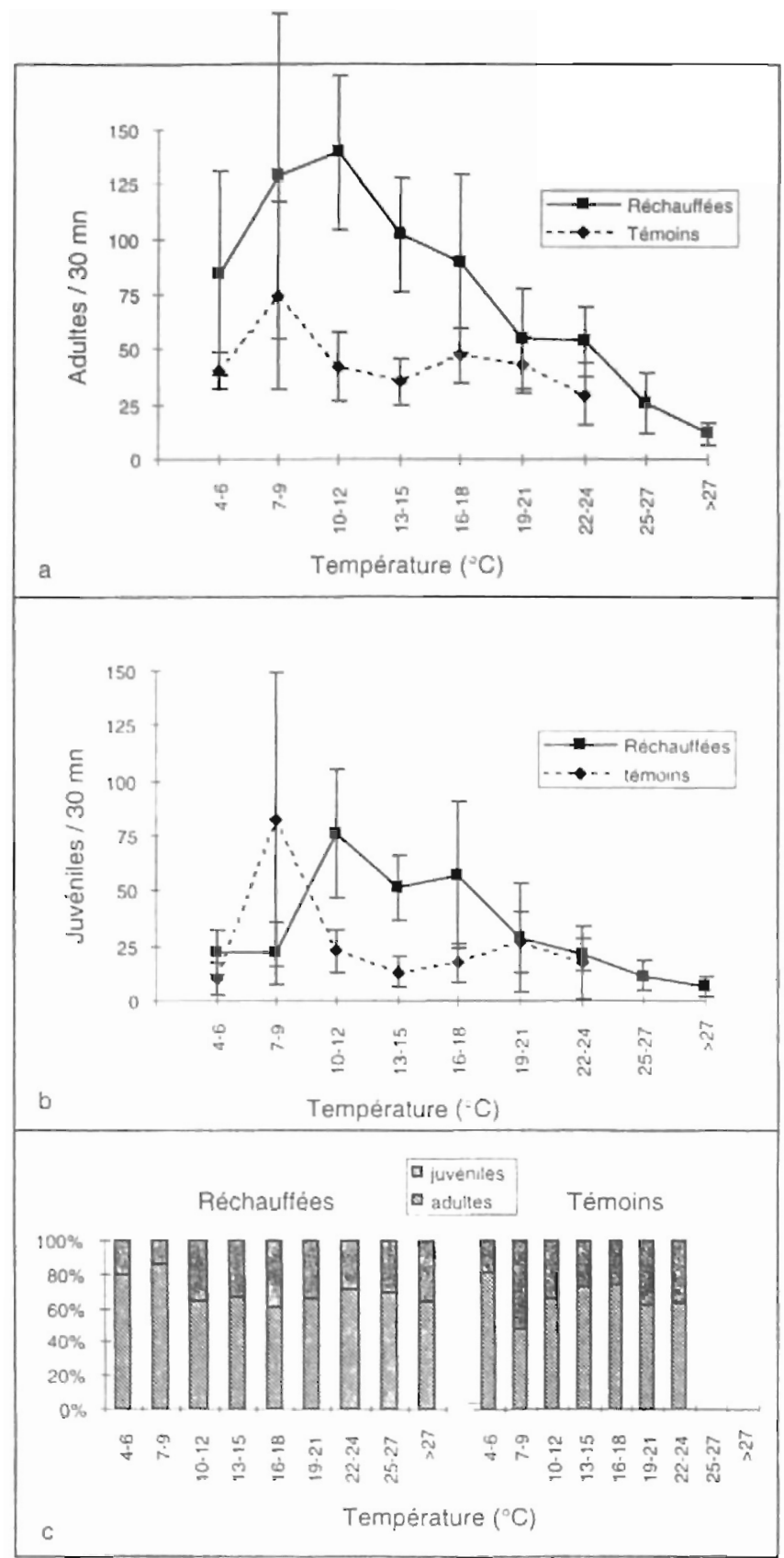

Fig. 4. - Résultats par groupes thermiques de $3^{\circ} \mathrm{C}$ d'amplitude, toutes campagnes confondues, sur les stations réchauffées (hors station 4 du rejet) et les stations témoin, avec intervalles de confiance à $95 \%$ (Student). a : effectifs d'adultes; b : effectifs de juvéniles; $c$ : contribution relative des adultes et des juvéniles.

Fig. 4. - Catches versus increasing classes of temperatures ( $3^{\circ} \mathrm{C}$ wide), when mixing all campaigns on heated sites (excepted station 4 directly located in the outflow of the powerplant) and control, with $95 \%$ Student confidence intervals. $a$ : adults number; $b$ : juveniles number; $c$ : adultes/juveniles ratio. 
La figure 5a, relative au nombre d'espèces (adultes plus juvéniles) ne montre qu'une baisse modérée, mais néanmoins significative, avec la température, et semble s'effondrer brutalement au dessus de $29^{\circ} \mathrm{C}$ (quatre pêches seulement à ces températures extrêmes). Les figures $5 b$ et $5 c$, qui rassemblent les données par groupes thermiques, montrent que le fléchissement du nombre d'espèces pêchées s'observe dès $25^{\circ} \mathrm{C}$ même en ne conservant que les $20 \%$ meilleures pêches (quintile supérieur) sur ce critère du nombre d'espèce pêchées.

Les espèces affichent des réponses contrastées devant l'élévation de température. La figure 6 est relative aux adultes. L'espèce la plus sensible est la truite: ses captures sont brutalement divisées par deux au dessus de $10^{\circ} \mathrm{C}$, pour décliner ensuite et s'annuler vers $25^{\circ} \mathrm{C}$. Les pêches réalisées à moins de $10^{\circ} \mathrm{C}$ correspondent cependant aux pêches d'hiver, saison où la dévalaison des truite excédentaires du bassin versant pourrait expliquer en partie ce net accroissement des captures. La vandoise est préférentiellement capturée au dessous de $16{ }^{\circ} \mathrm{C}$ puis ses captures baissent progressivement pour s'annuler autour de $25^{\circ} \mathrm{C}$. Le blageon semble suivre un même schéma mais le faible nombre d'individus pêchés entache la courbe d'une forte incertitude. Le chevaine montre un optimum entre 7 et $13^{\circ} \mathrm{C}$, ses captures sont importantes et stables jusqu'à $25^{\circ} \mathrm{C}$, et il reste présent à plus de $27^{\circ} \mathrm{C}$. Le barbeau fluviatile et le hotu affichent un peu le même type de courbe que le chevaine, mais disparaissent pratiquement au dessus de $25^{\circ} \mathrm{C}$. Rappelons que ces deux dernières espèces sont plutôt inféodées au chenal et assez mal représentées par des pêches de bordure. Les captures de gardon et de brochet ne déclinent qu'assez tard $\left(22^{\circ} \mathrm{C}\right.$ pour le premier, $24^{\circ} \mathrm{C}$ pour le second), mais elles s'annulent vers $27^{\circ} \mathrm{C}$. Le spirlin affiche une courbe en cloche, assez comparable à celle du chevaine, mais décalée vers les hautes températures: il n'est pas pris à moins de $6^{\circ} \mathrm{C}$ ou à plus de $27^{\circ} \mathrm{C}$, avec un maximum entre 10 et $16^{\circ} \mathrm{C}$ et un palier encore assez haut entre 16 et $25^{\circ} \mathrm{C}$. Les captures de goujon sont relativement stables sur une très large gamme de température $\left(7\right.$ à $\left.27^{\circ} \mathrm{C}\right)$, mais sont pratiquement nulles hors de cette gamme. La perche commune est capturée sur toute la gamme thermique, de $4^{\circ} \mathrm{C}$ à plus de $27^{\circ} \mathrm{C}$, avec un net optimum entre 13 et $21^{\circ} \mathrm{C}$. L'ablette et la perche soleil ne montrent pratiquement pas d'évolution des captures avec la température, ces dernières se maintiennent même à plus de $27^{\circ} \mathrm{C}$. La brême bordelière est trop peu capturée pour que sa courbe soit fiable, mais semble, comme l'ablette et la perche soleil, tolérante à toute la gamme thermique.

La figure 7 est relative aux juvéniles. Les courbes sont plus incertaines dans la mesure où les effectifs sont moitié moins importants que pour les adultes, et beaucoup plus variables d'une pêche à l'autre. Les courbes ressemblent globalement à celles des adultes à l'exception nota- 


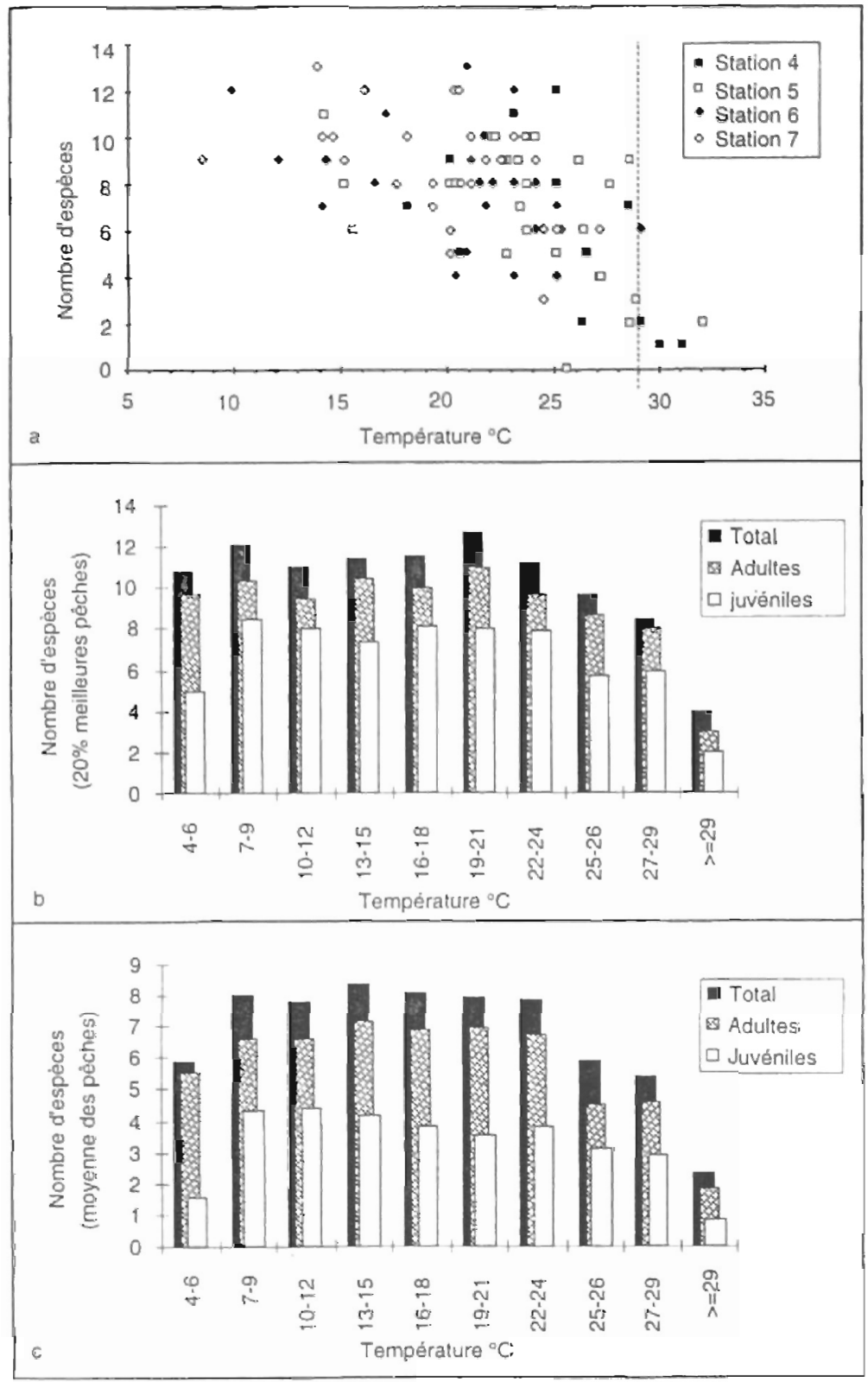

Fig. 5. - Richesse spécifique. a: Nombre d'espèces capturées sur les stations réchauffées en période estivale (avril à septembre); $b$ : contribution relative des adultes et des juvéniles, toutes campagnes et toutes stations confondues, en ne gardant que la moyenne des $20 \%$ meilleures pêches sur ce critère de la richesse spécifique; $c$ : idem b. mais les valeurs moyennes portent sur l'ensemble des pêches.

Fig. 5. - Number of fish species. a : number of speacies catched during each fishing on heated stations during the warm season (april to september); $b$ : relative importance of adultes and juveniles when mixing all campaigns and stations, when keeping the $20 \%$ best fishing campaigns on the fish diversity criterion; $c$ : as fig. $5 b$, but using all the data. 


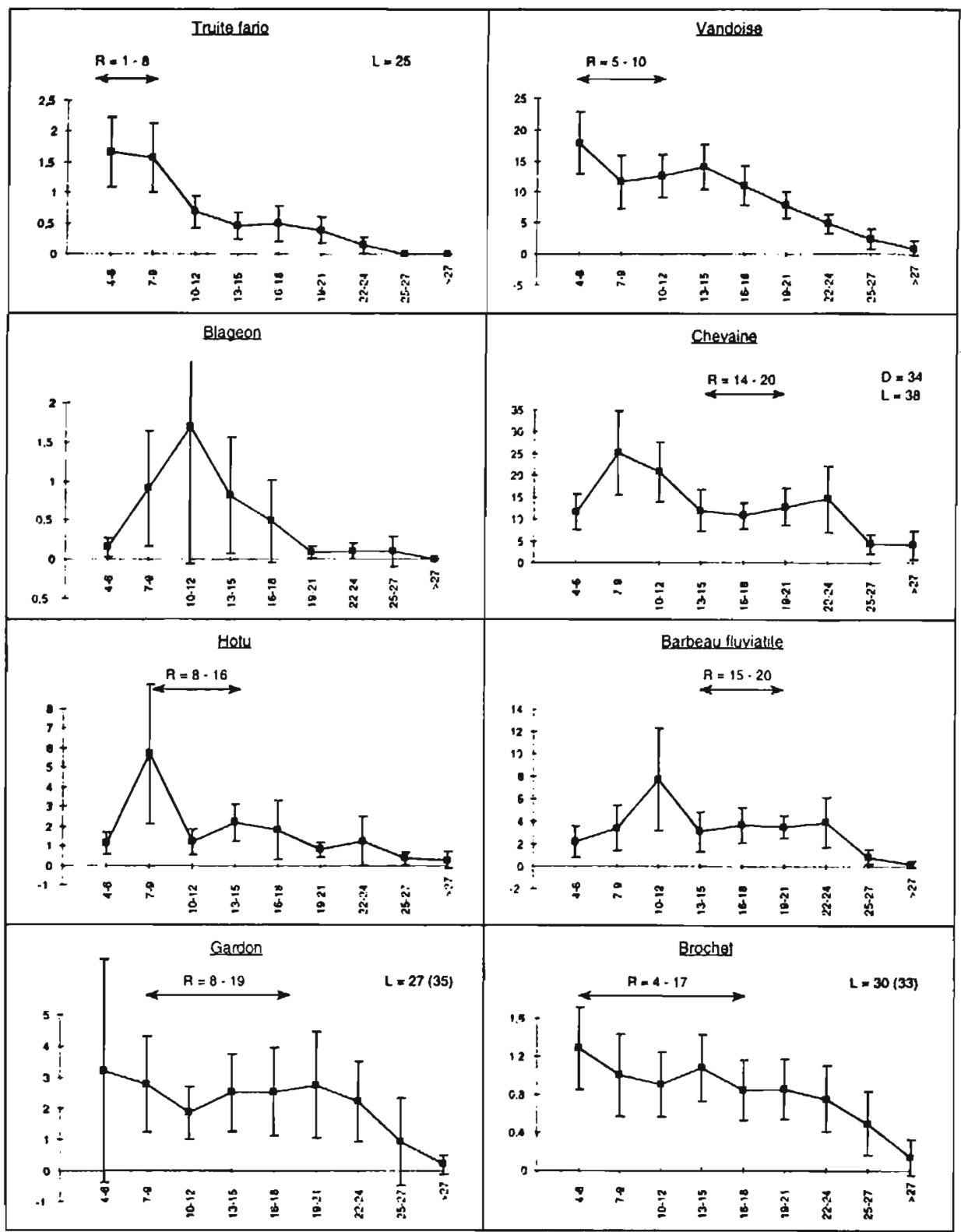




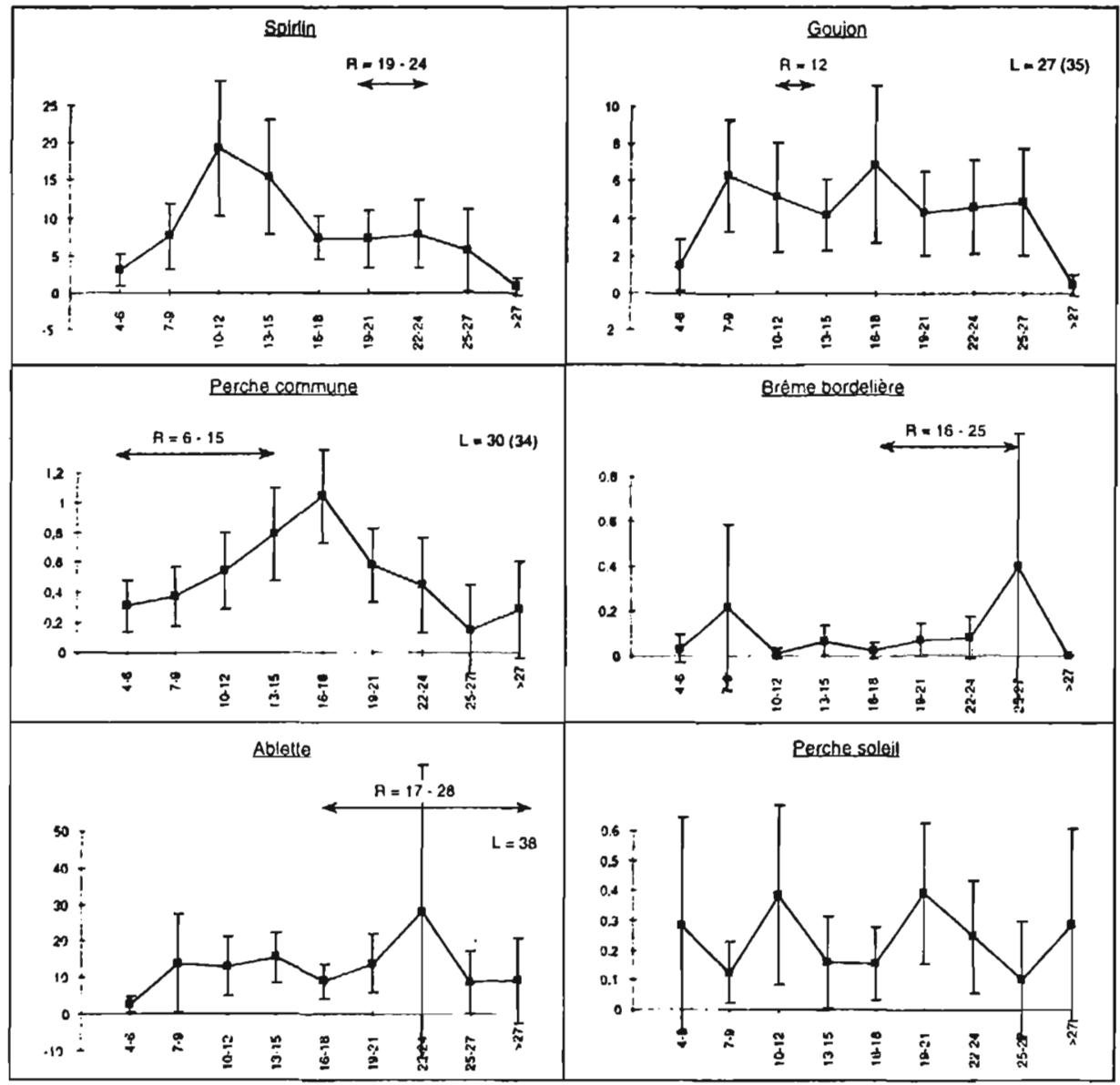

Fig. 6. - Espèce par espèce, toutes stalions et campagnes confondues, effectifs moyens d'adultes pêchés en fonction de la température, avec intervalles de confiance à $95 \%$ sur la moyenne (Student). Lorsque les données sont disponibles (Alabaster et Lloyd, 1982), indication de $\mathrm{A}$ la gamme thermique de reproduction, et $L$ la température létale. La lempérature entre parenthèse indique la température létale après adaptation progressive aux hautes températures.

Fig. 6. - Using all the data, for each species, mean catches of adults versus temperature with $95 \%$ confidence interval. $R$ is the spawning thermal range, $L$ the letal temperature, after Alabaster and Lloyd, 1982. In parenthesis is the letal temperature after previous thermal adaptation.

ble de la vandoise (et dans une moindre mesure du hotu et du barbeau) dont les juvéniles semblent à la fois mieux résister aux hautes températures et être moins pêchés aux basses températures.
Remarque : L'efficacité de la pêche électrique varie avec la température (Philippart, 1979). Une température trop basse $\left(<5^{\circ} \mathrm{C}\right)$ est néfaste, du fait de la léthargie du poisson. A l'opposé une température élevée (sans doute 


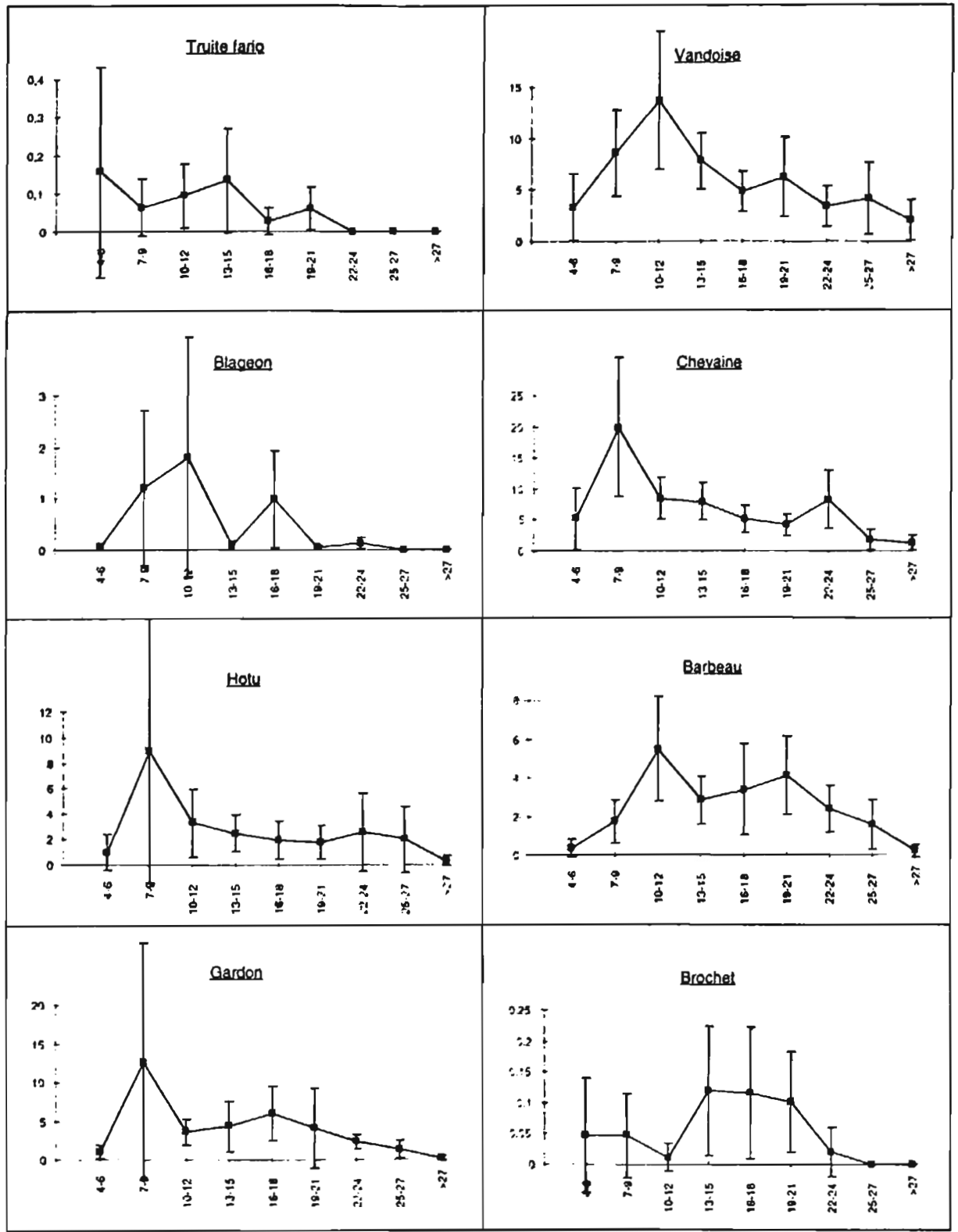




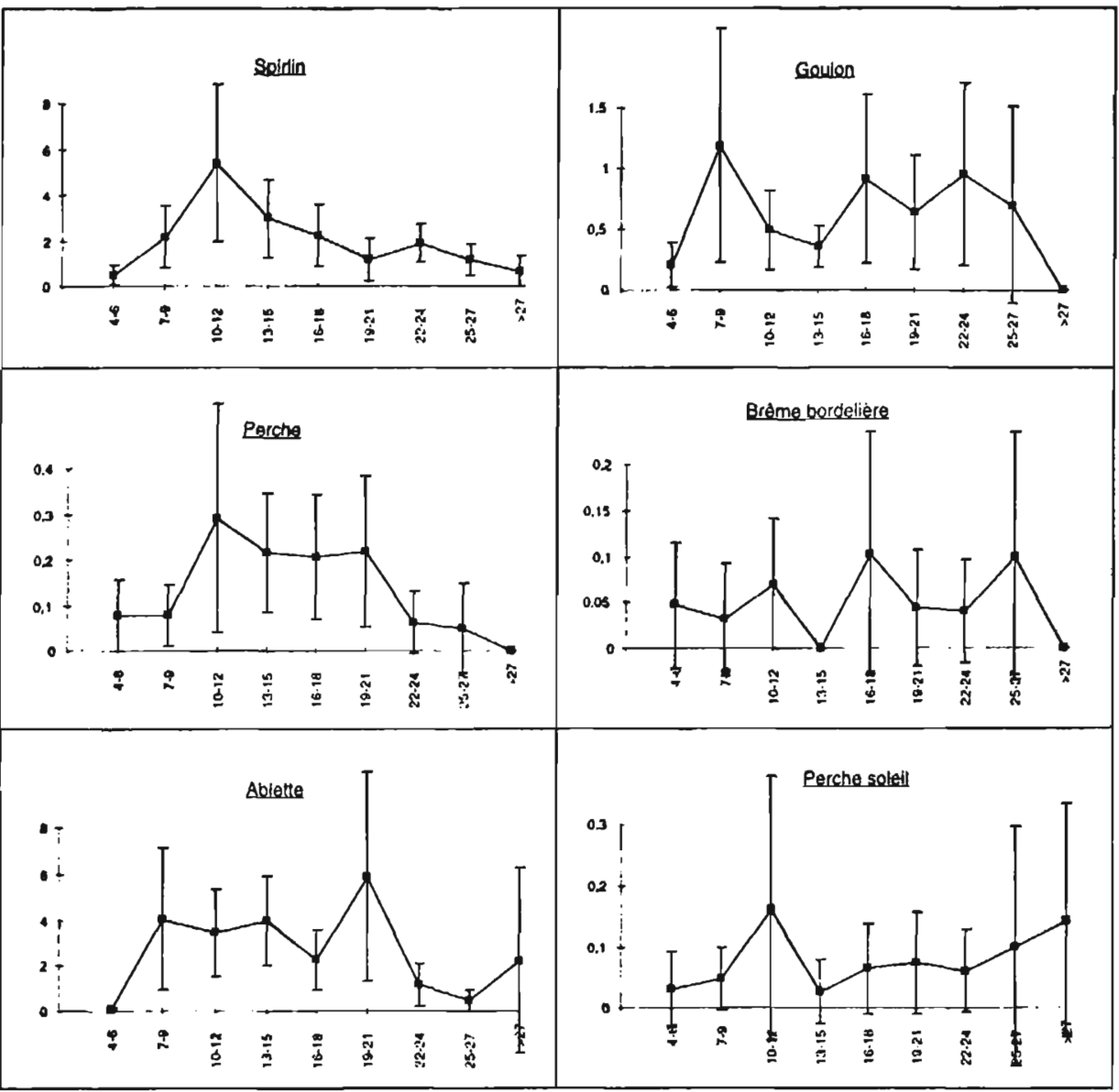

Fig. 7. - Effectifs moyens de juvéniles (cf. fig. 6). Les intervalles de confiance sont donnés à titre indicatif, la répartition des juvéniles n'étant pas normale autour de la moyenne, mais avec un très fort pourcentage de pèches nulles $(17 \%)$.

Fig. 7. - As fig. 6, for juveniles. Student confidence intervals are just for information since juveniles catches do not follow a normal distribution but show a high percentage of nulls (17\% versus $0.6 \%$ for adults).

dès $20^{\circ} \mathrm{C}$ ) l'est aussi, car elle augmente tout à la fois le métabolisme du poisson et donc sa tendance à la fuite, ainsi que sa résistance à la galvano-narcose. Ces effets sont très difficiles à quantifier in situ, mais les résultats obtenus ici sont différenciés d'une espèce à l'autre et en accord avec leur biologie. II ne semble donc pas que la possible altération des pêches avec la température amène un biais sensible sur nos données, sauf pour les températures les plus basses. 


\subsection{Effet quantitatif de l'écart thermique entre stations en période hivernale}

L'objectif est de déceler s'il existe une relation entre les effectifs pêchés sur les stations réchauffées et l'amplitude thermique entre ces stations et les stations amont. Cette relation est recherchée en période hivernale, pour des températures inférieures à $23^{\circ} \mathrm{C}$, ceci pour rester dans une gamme où l'effet "niveau thermique" que nous venons d'étudier n'est pas encore trop sensible. Il est en effet impossible de dissocier totalement l'effet "niveau thermique" de l'effet "différentiel" car les forts écarts thermiques sont nécessairement associés à des températures plutôt chaudes, même en hiver. Des tests de linéarité effectués sur les observations brutes sur chacune des stations ne montrent aucune corrélation positive entre écart thermique et résultat des pêches, que ce dernier soit exprimé en biomasses, en effectifs d'adulte, ou en effectifs de juvéniles. Et un test global, toutes stations réchauffées confondues et en normant les effectifs par la moyenne de chaque station pour les rendre comparables, ne montre toujours aucun lien entre effectifs et amplitudes thermiques. Cette absence de corrélation, avec des tendances même plutôt opposées d'une station à l'autre apparaît bien sur la représentation de la moyenne des biomasses et des effectifs d'adultes par classe d'écart thermique (fig. 8). Pour détecter d'éventuelles migrations entre la station 6 et la station $6 \mathrm{G}$ située en face et non réchauffée, nous avons porté sur cette figure les résultats des pêches de la station 6G correspondants aux écarts thermiques de la station 6 , en excluant la dernière classe d'écart thermique $\left(8-10^{\circ} \mathrm{C}\right)$ qui ne comprend que trois observations. Une corrélation négative serait apparue entre les stations 6 et $6 G$ si l'écart thermique entre ces stations avait avantagé la première, ce qui n'est pas le cas.

En définitive, et contrairement à ce que l'on aurait pu penser, l'attrait hivernal des poissons vers les veines chaudes semble quantitativement négligeable, et l'on ne décèle pas de déplacements de la station 6G non réchauffée vers la station 6 située en face et réchauffée.

\section{ASPECTS STRUCTURAUX}

L'enjeu est de déterminer si le réchauffement joue sur la composition spécifique des pêches. Treize espèces ont été analysées (tableau 1): onze dont les effectifs dépassent $1 \%$ du total des captures d'adultes, plus la perche commune $(0,9 \%$ des captures), et la perche soleil $(0,4 \%)$ car ces deux espèces sont susceptibles d'être mieux représentées dans les zones réchauffées. Afin de limiter l'effet taille, tant sur les espèces (le chevaine est plus représenté que la truite) que sur les pêches (certaines pêches sont meilleures que d'autres), et ne garder que les aspects structuraux du peuplement, nous effectuerons des analyses factorielles des correspondances (AFC). Leur particularité 


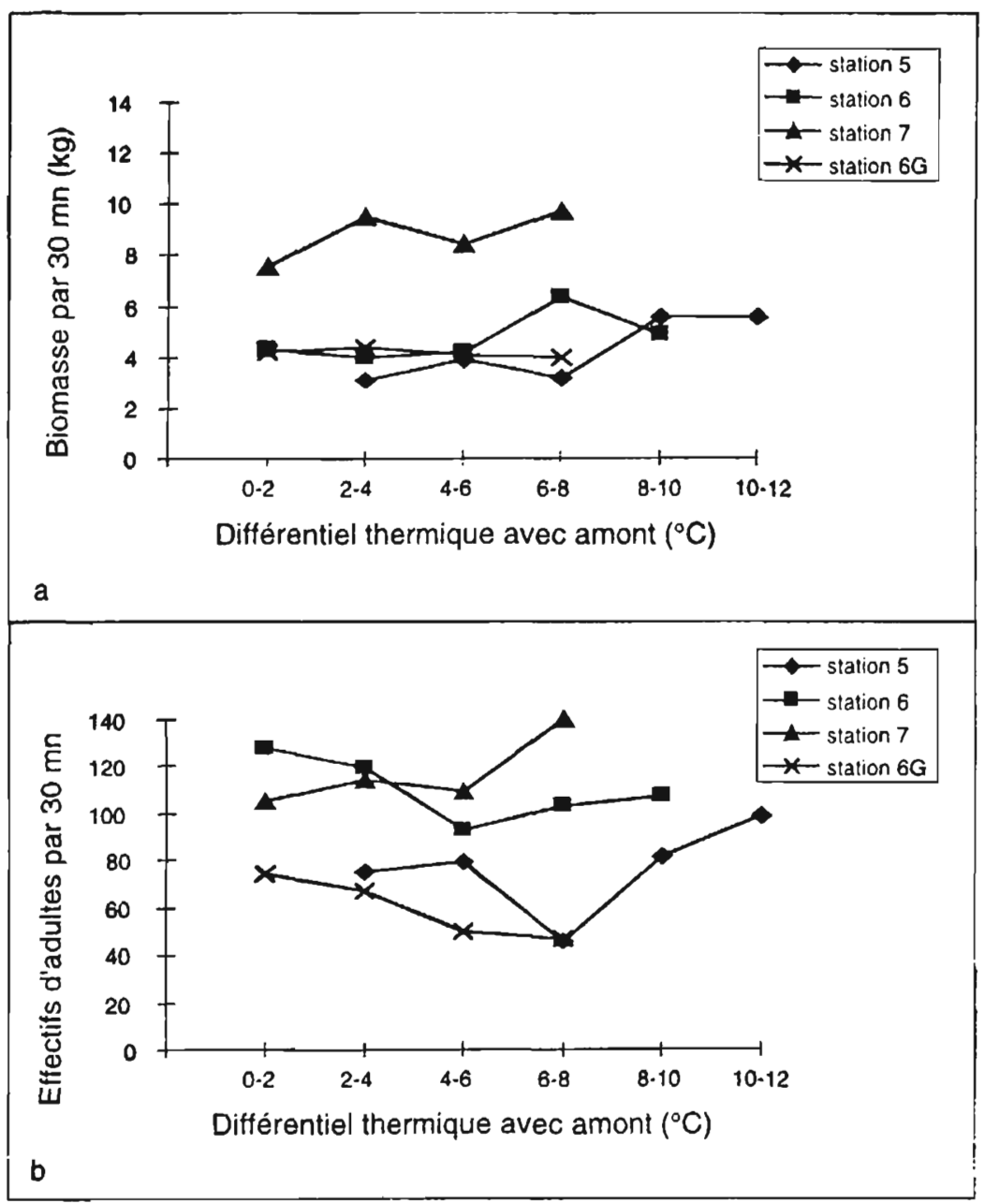

Fig. 8. - Résultats des pêches sur les stations réchauffées en fonction de l'écart thermique avec les stations amont. $\mathrm{a}$ : biomasses; $\mathrm{b}$ : effectifs d'adultes. Les écarts thermiques de la station non réchauffée $6 \mathrm{G}$ sont ceux de la station 6 , pour étudier une éventuelle migration entre ces deux stations.

Fig. 8. - Catches on heated stations versus thermal differential with control stations, expressed in biomasses (a) and adults number (b). Thermal data of station $6 G$ are those of station 6 in order to study possible migration between the two stations.

est de travailler sur les distributions de fréquences et non directement sur les effectifs. Par ailleurs le logarithme (base 10) des effectifs a été utilisé pour limiter le poids des pêches exceptionnelles. Les analyses et les graphiques qui s'y rapportent ont été effectués à l'aide des modules d'Ana- 
lyse de Données Ecologiques (ADE) développés par l'Université Lyon-1 (Thioulouse et al., 1995).

\subsection{Structure liée au niveau thermique}

La structure de la composition des pêches en fonction de la température est recherchée sur l'ensemble des campagnes et des stations. Celles-ci sont ordonnées par températures croissantes (de 4 à $32^{\circ} \mathrm{C}$ ), et rassemblées par groupes de $3^{\circ} \mathrm{C}$ afin d'obtenir des effectifs suffisants pour avoir un comportement stable. Ce type d'analyse, dite "inter-groupe", compare la moyenne des profils de chaque groupe. On gomme ainsi les effets autres que thermiques puisque qu'ils sont répartis équitablement dans tous les groupes, à conditions d'être indépendants de l'effet thermique. Nous cherchons en particulier ici à gommer l'effet station (mélange de toutes les stations pour un groupe de température donnée) ou l'effet année (l'évolution temporelle sur les 16 ans d'étude qui ne sera pas abordée ici). Dans la pratique, l'élimination de l'effet station est incomplet puisque les groupes des hautes températures comportent surtout des stations réchauffées. II nous faudra donc discuter de la représentativité des stations vis-à-vis de ces aspects structuraux.

Une première analyse est effectuée sur les effectifs d'adultes. Son résumé est donné figure 9a. L'essentiel de la structure est porté par l'axe F1 comme le confirme le graphe des va- leurs propres. L'axe F1 est nécessairement thermique du fait de la constitution des groupes. II explique $56 \%$ de la variance entre groupes thermiques. Le reste est pour l'essentiel un bruit de fond dû à la grande variabilité des pêches, sensible malgré la grande taille de nos groupes. Le plan F1-F2 montre 3 amas séparés par leur position moyenne et par la forme de leur ellipse d'inertie. Le groupe thermique 1 $\left(4\right.$ à $6^{\circ} \mathrm{C}$ ) et les groupes thermiques 8 $\left(25-27^{\circ} \mathrm{C}\right)$ et $9\left(>27^{\circ} \mathrm{C}\right)$ s'opposent au groupe central au sein duquel l'ordination est néanmoins respectée, hormis une inversion entre les groupes centraux $4\left(13-15^{\circ} \mathrm{C}\right)$ et $5\left(16-18^{\circ} \mathrm{C}\right)$. Cette analyse intergroupes n'explique que $10 \%$ de la variance totale des observations malgré un découpage assez fin du gradient thermique. Si l'effet thermique est réel, la pêche moyenne qu'il permet de prédire n'est bien qu'une moyenne et la variabilité des pêches (visualisée par la grande taille des ellipses) est très grande.

Le plan F1-F2 de la même analyse faite sur les juvéniles ne présente aucune structure (fig. 9b): les profils thermiques moyens se ressemblent, ou plus exactement deux profils appartenant à deux groupes thermiques éloignés peuvent autant se ressembler que deux profils appartenant à des groupes voisins. La figure $10 \mathrm{~b}$ permet de le visualiser: le groupe 1, avec une forte proportion de chevaine ressemble autant au groupe 7 qu'au groupe 2 , et le groupe 3 , avec une forte proportion de vandoise, ressemble autant au 8 qu'au 4. Cette absence de structure se maintient même 


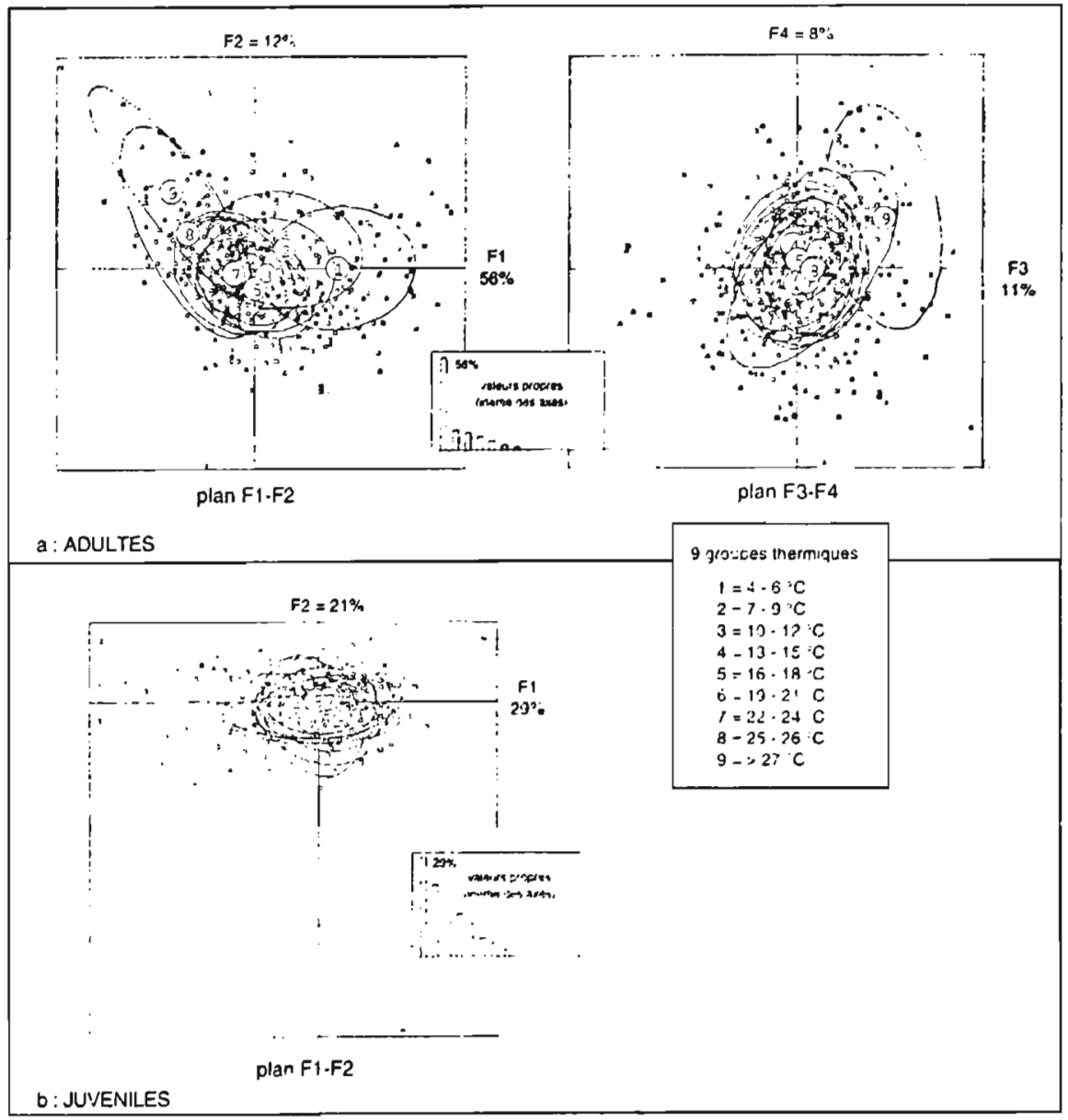

Fig. 9. - Résumé de l'AFC inter-groupes thermiques sur le logarithme des effectifs d'adultes (a) et de juvéniles (b), toutes stations et toutes campagnes confondues. Les ellipses regroupent $50 \%$ des observations visualisées par de petits carrés.

Fig. 9. - Summary of the inter-thermal groups COA on (the logarithm of) adults number (a) and juveniles number (b), using the data of all campaigns and stations (519 fishing parties). Ellipses contain $50 \%$ of the data of a thermal group.

en limitant l'effet des pêches exceptionnelles en utilisant des classes d'effectifs ( 5 classes) à la place de la transformation logarithmique des effectifs, ou en ne conservant que les pêches comportant au moins trois espèces. II n'y a donc pas de lien sen- sible entre la température et la structure des pêches de juvéniles dans la gamme thermique obsenée, la plupart des espèces peuvent se retrouver en grande proportion dans un groupe thermique froid comme dans un groupe thermique chaud. 

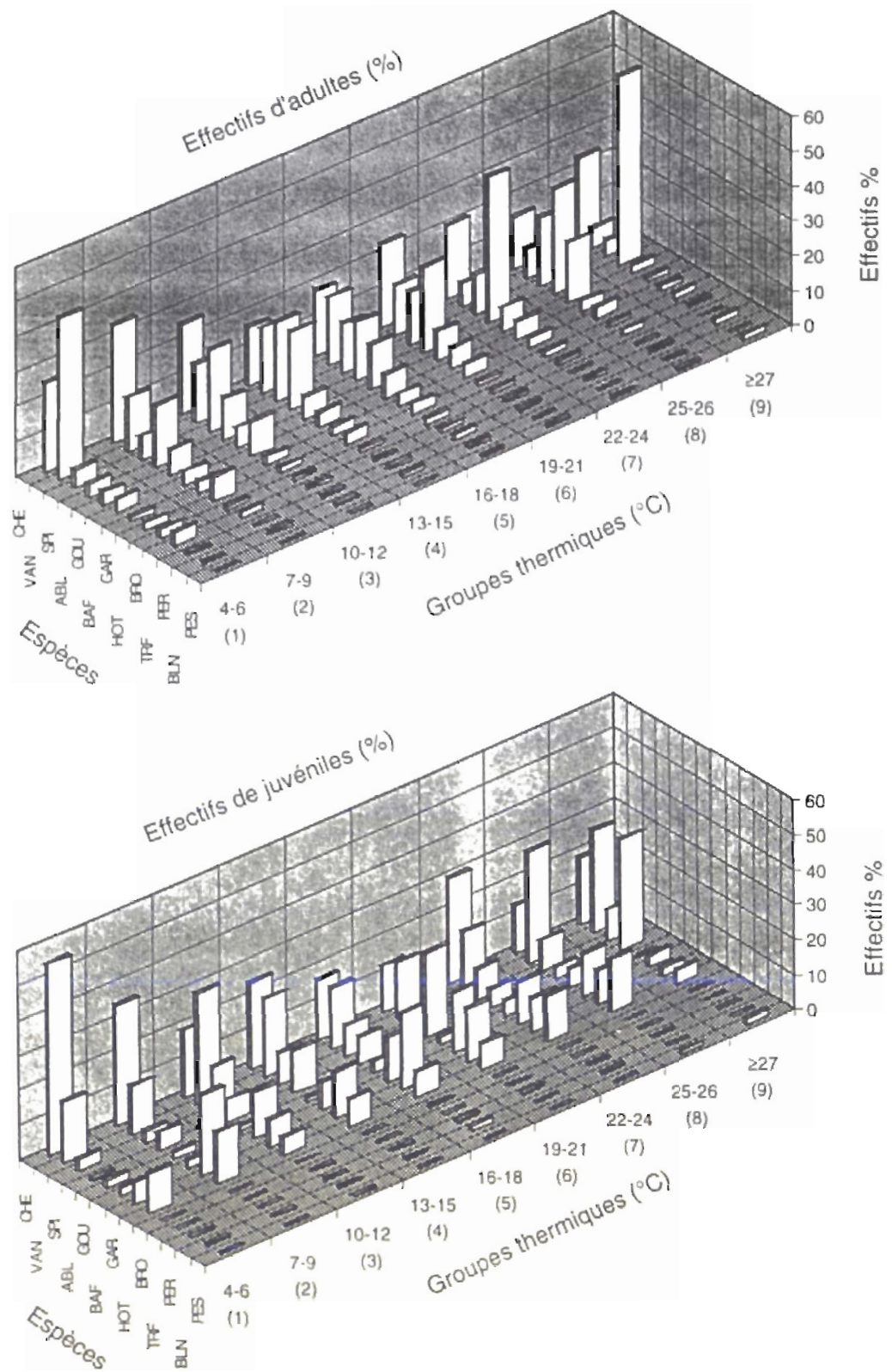

Fig. 10. - Profils des pêches en fonction de la température, toutes campagnes et toutes stations confondues. $a$ : adultes; $b$ : juvéniles.

Fig. 10. - Relative catches patterns versus temperature, mixing all campaigns and stations, for adults (a), and juveniles (b). 


\subsection{Représentativité des stations}

Pour tester l'influence des stations de pêche sur la structure observée, un même type d'analyse est effectué en groupant les pêches par station. Les résultats sont résumés sur la figure 11. L'effet station s'exprime sur les trois premiers axes factoriels (graphe des valeurs propres). Les trois stations amont sont regroupées (la 1 cachée par la 2) avec la station 6G. Elles remplissent donc bien leur rôle de témoins, malgré leurs différences morphologiques. Les trois stations réchauffées 5,6 et 7 sont également regroupées et s'individualisent des stations témoins. On notera ce regroupement malgré le gradient thermique décroissant de la station 5 vers la station 7. Ceci est à rapprocher de l'analyse précédente, la structure des pèches évolue peu dans la gamme thermique centrale. La station 4 , située dans le canal de rejet, présente des caractéristiques thermiques extrêmes et apparaît isolée. Elle est à elle seule responsable de la structure observée sur les deux axes factoriels suivants. La station 8 est également isolée, différente des stations témoins. Supposée représenter les conditions du Rhône après mélange complet des eaux, elle est visiblement trop soumise à l'influence faunistique et hydraulique de l'Ain pour jouer ce rôle.

II ressort de ces deux analyses, mis à part pour la station 8 , que les stations se différencient plus du fait de

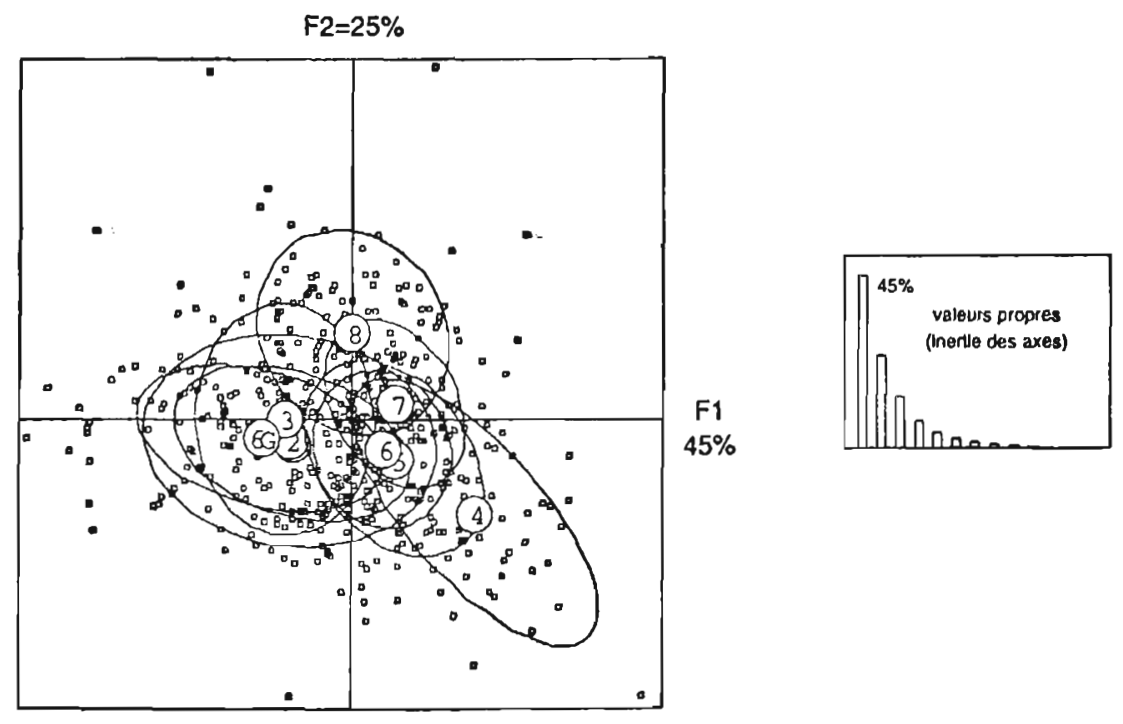

Fig. 11. - Résumé de l'AFC «inter-stations ». Même conditions que figure 9. Les ellipse regroupent $90 \%$ des observations.

Fig. 11. - Summary of the inter-stations COA (as fig. 9). Ellipses include $90 \%$ of the data of a station. 
leurs régimes thermique que de leurs differrences morphologiques. Les stations se regroupent selon leurs classes thermiques et non selon leur morphologie, alors que sur ce critère les trois stations amont sont très différentes, avec en particulier une station 3 morphologiquement assez proche de la 7, et la 1 assez proche de la 6 . Ceci nous autorise à considérer que les stations sont comparables sur le plan de la structure des pêches (ce qui n'est pas le cas quantitativement, les stations réchauffées ayant globalement un habitat plus favorable, cf. $\S 3.1$ ), et que l'effet station peut donc être négligé dans la suite des analyses structurelles.

\subsection{Analyse structurelle des pêches (adultes)}

Une AFC sans contrainte (sans imposer de groupes a priori) du tableau précédent des effectifs d'adultes laisse 456 pêches en écartant la station 8 (fig. 12). L'histogramme de l'inertie des axes affiche une décroissance lente, reflet d'une grande indépendance des variables (les 13 espèces de poissons). Chaque poisson a un comportement peu lié à celui de ses voisins, et il existe peu d'associations (corrélations) entre poissons permettant de diminuer la dimension du tableau de données.

Le premier plan exprime $30 \%$ de la variance totale, à comparer à une valeur seuil de $15 \%$ si les 13 espèces avaient un comportement aléatoire. II est créé par 6 espèces, dans l'ordre d'importance décroissante l'ablette (axes 1 et 2), le goujon (axe 2), la vandoise (axes 1 et 2), la truite (axe 1), le brochet (axe 2), et le spirlin (axe 1), auxquelles s'ajoutent 2 espèces à contribution plus faible, le chevaine (axes 1 et 2) et la perche soleil (axe 2). Soit 8 espèces sur 13 bien ou assez bien représentées (fig. 12c). Les barycentres des 9 groupes thermiques se projettent sur le premier axe de manière quasiment identique à la première analyse (fig. 12a). Le premier facteur influençant la structure des pêches est bien le facteur thermique, opposant des pêches à forte proportion de truite et de vandoise aux pêches associant le couple ablette-spirlin. En projettant la position moyenne des pêches regroupées seIon 4 classes de débits (moins de $350 \mathrm{~m}^{3} / \mathrm{s}, \quad 350$ à $550 \mathrm{~m}^{3} / \mathrm{s}, \quad 550$ à $750 \mathrm{~m}^{3} / \mathrm{s}$, plus de $750 \mathrm{~m}^{3} / \mathrm{s}$ ), un échelonnement régulier sur l'axe 2 apparaît, opposant notamment le goujon et la perche soleil à faible débit au brochet et à l'ablette aux forts débits (figure 12b). Le deuxième facteur structurant les pêches apparaît donc être le débit. L'axe 3 ne semble pas interprétable. II est caractérisé par des pêches importantes de truite (jusqu'à 10 adultes), de brochet (jusqu'à 8 unités), ou de perche (jusqu'à 6 unités) sans que l'on puisse relier ces différentes pêches. Les axes suivants procèdent de même avec d'autres espèces, ce qui illustre le fait qu'une bonne part de la variabilité des données est due à des pêches un peu particulières et sans doute assez aléatoires qui fait qu'on obtient presque autant d'axes factoriels indépen- 

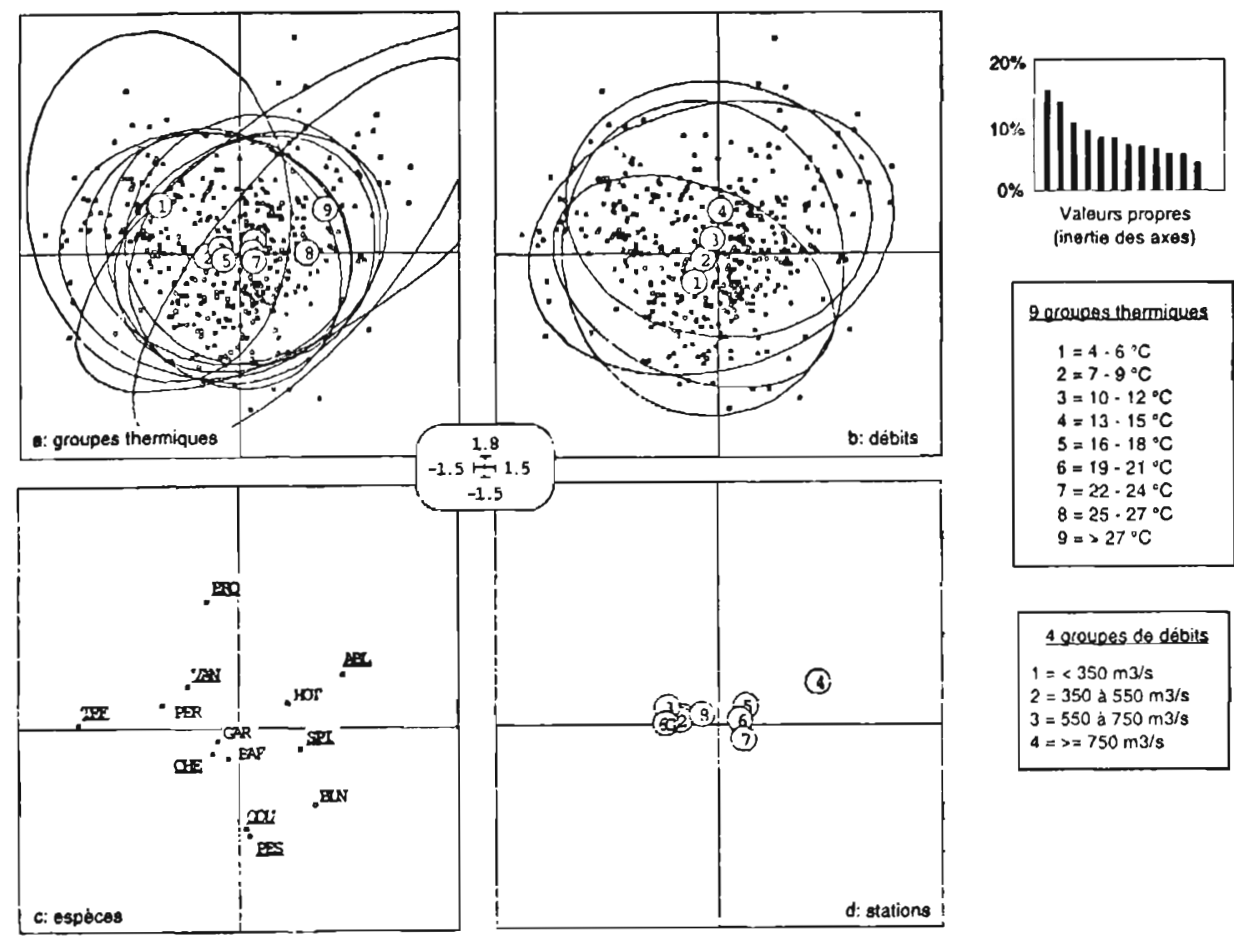

2 areupes themiques

$1=4 \cdot 6^{\circ} \mathrm{C}$

$2=7 \cdot 9^{\circ} \mathrm{C}$

$3=10 \cdot 12^{\circ} \mathrm{C}$

$4=13 \cdot 15^{\circ} \mathrm{C}$

$5=16-18^{\circ} \mathrm{C}$

$6=19-21^{\circ} \mathrm{C}$

$7=22 \cdot 24^{\circ} \mathrm{C}$

$8=25 \cdot 27^{\circ} \mathrm{C}$

$9=>27^{\circ} \mathrm{C}$

4 groupes de débits

$1=<350 \mathrm{~m} 3 / \mathrm{s}$

$2=350$ d $550 \mathrm{~m} 3 / \mathrm{s}$

$3=550 \mathrm{a} 750 \mathrm{~m} 3 / \mathrm{s}$

$4=>=750 \mathrm{~m} 3 / \mathrm{s}$

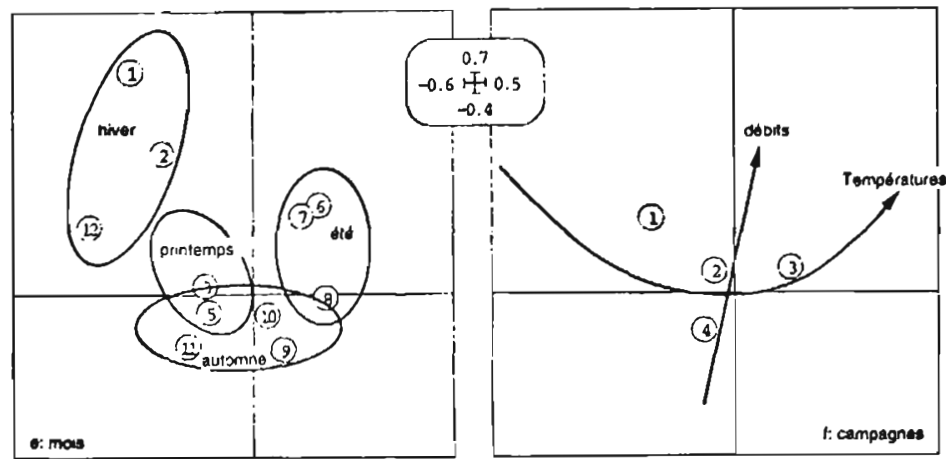

\begin{tabular}{|c|c|c|}
\hline Mas & \multicolumn{2}{|c|}{ "C débils } \\
\hline 1: janver & 8.2 & 585 \\
\hline 2: IAvnar & B.2 & . \\
\hline 3: mass & 9,7 & 480 \\
\hline 5: mai & 16,6 & 590 \\
\hline 6. pun & 16.6 & 690 \\
\hline 7. jull:et & 22,3 & 500 \\
\hline Q: aov: & 21,9 & 450 \\
\hline F seotembre & 20.5 & 470 \\
\hline in oceoro & 16.6 & 313 \\
\hline 11: revertivg & 11,6 & 513 \\
\hline 12 tecembre & 8.6 & 242 \\
\hline Camcazng 1. & 9.1 & 470 \\
\hline Camoange 2 : & 16,3 & 604 \\
\hline Camoagre 3 & 21.8 & 503 \\
\hline Campagre $4:$ & 13,8 & 450 \\
\hline
\end{tabular}

Fig. 12. - Résumé de l'AFC sans contrainte sur les effectifs d'adultes, toutes stations (sauf 8 ) et toutes campagnes confondues. Rhône, secleur du Bugey, années 1979-1994, 456 pêches. a: projection des pêches par groupes thermiques; $b$ : projection des pêches par groupes de débits; $c$ : projection des espèces (variables de l'analyse), sont soulignées les espèces à forte contribution au plan; $d$ : projection des pêches par stations, station 8 en individu supplémentaire; e : projection des pêches regroupées par mois et f par campagnes. Les ellipses regroupent $90 \%$ des observations de chaque groupe.

Fig. 12. - Summary of an unconstrained COA on adults number using data of all campaigns and stations excepted station 8 (456 fishing parties). a : projection of catches gathered by thermal groups; $\mathrm{b}$ : projection of calches gathered by increasing discharge groups; $c$ : projection of species (the variables of the COA). Species with a high contribution to the plane are underlined; $d$ : projection of the catches gathered by sites (station 8 is a supplementary variable); e : projection of catches gathered by monthes, and $f$ : gathered by campaigns. Ellipses include $90 \%$ of the data of each group. 
dants que d'espèces de poissons. Seul le premier plan apparaît donc interprétable. Les stations se projettent sur ce plan d'une manière comparable à l'analyse précédente (figure 12d), à l'exception de la station 8 (projetée en individu supplémentaire car ne participant pas à l'analyse), moins individualisée et située à mi-chemin des stations témoins et réchauffées. D'où l'importance d'effectuer conjointement des analyses spécifiques intergroupes si l'on cherche à maximiser la distance entre des groupes donnés.

Les deux derniers graphes (fig. 12e et $12 f$, échelle dilatée) donnent la projection des campagnes et des mois de pêche (de 15 à 79 observations par mois, le mois d'avril avec 6 observations est supprimé). Les ordinations sur la température et le débit ne sont pas toujours bien respectées. Le nombre d'observations étant suffisant pour avoir des images stables sur ce premier plan factoriel, cela signifie qu'il existe probablement un effet saisonnier qui se surimpose aux effets thermiques et débits. En particulier, les pêches du mois de juin (32 observations) rejoignent déjà la structure des pêches du mois de juillet (58 observations) malgré une forte différence de température et de débit, et se séparent des pêches de mai 72 observations) avec lesquelles elles partagent pourtant la même température moyenne de $16,6^{\circ} \mathrm{C}$. De même les pêches du mois de mars (79 observations) se rapprochent des pêches de mai malgré un fort écart thermique. Enfin, les pêches d'août (39 observations) et septembre (21 observations) affichent des positions voisines sur l'axe 1, en relation avec des températures élevées et proches, mais différentes sur l'axe 2 alors que leurs débits moyens sont proches. Tout se passe comme si la structure des pêches du mois d'août anticipait la baisse automnale des débits. En contraste le retour aux conditions froides depuis la fin de l'été montre un gradient régulier en accord avec les conditions de températures et de débits.

\subsection{Quelques pêches types}

Les analyses structurelles sur les adultes peuvent être résumées par la figure 13 des conditions moyennes de température et de débit pour la capture de chaque espèce, et par la figure 14 de la composition des trois principaux groupes thermiques.

Le groupe froid $\left(4-6^{\circ} \mathrm{C}\right)$ montre une nette dominance de la vandoise ( $45 \%$ des effectifs) suivie du chevaine $(25 \%)$. La truite, avec plus de $4 \%$ est bien représentée et arrive en quatrième position derrière le spirlin. On peut néanmoins se demander si la composition spécifique particulière de ce groupe froid ne tient pas autant à la moindre capturabilité des autres espèces aux basses températures qu'à leur absence des bordures.

Le groupe central, très large (7$24^{\circ} \mathrm{C}$ ), représente la pêche moyenne sur ce secteur du Rhône: le chevaine, la vandoise, l'ablette et le spirlin se partagent à part sensiblement égale $80 \%$ des effectifs d'adultes. 


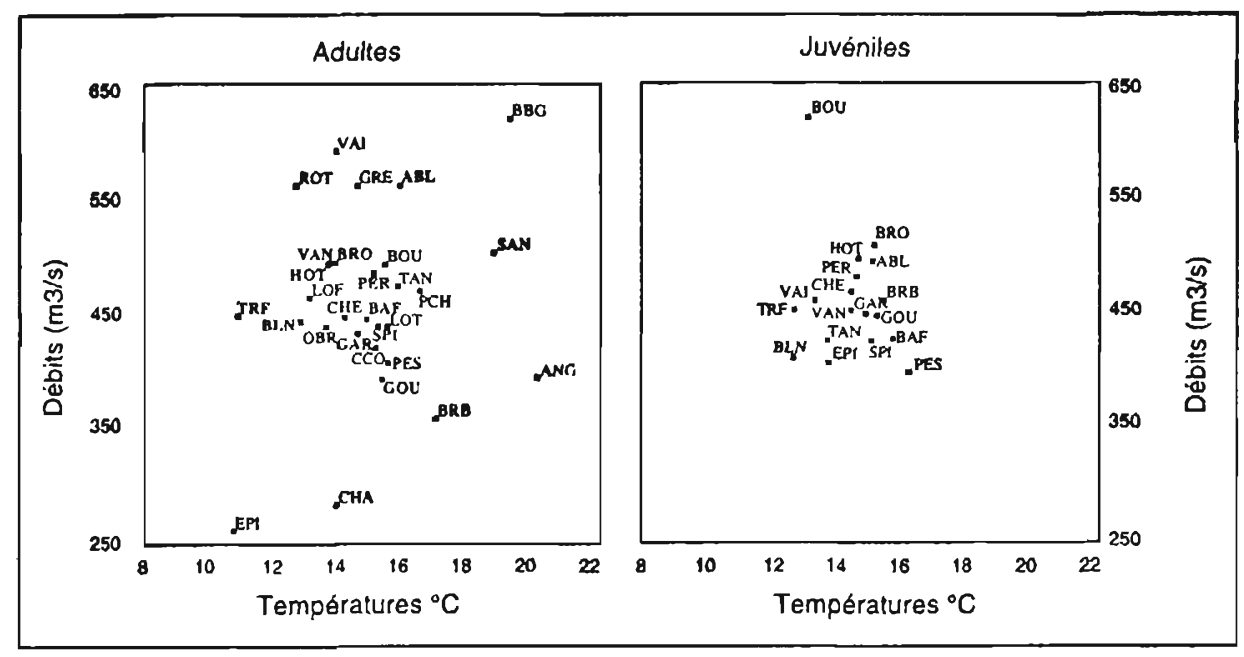

Fig. 13. - Cartographie des espèces en fonction de la température et du débit. Chaque position est obtenue en pondérant les coordonnées par le logarithme décimal des effectifs des pêches. Sont représentées les espèces pêchées au moins à quatre reprises, et en gras les 13 espèces ayant participé aux analyses.

Fig. 13. - Mean position of species versus temperature and discharge. Coordinates are weighed by the $\log 10$ of the fish number. Only species catched at least 4 times are plotted, and the species used in the COA analysis are plotted in bold.

Bien que hotu et barbeau soient sousreprésentés par ces pêches de bordure, le peuplement est encore typique de ce secteur du Rhône, quoique la vandoise soit sous-représentée. La composition de ce groupe se modife avec le débit. A faible débit $\left(\mathrm{m}^{3} / \mathrm{s}\right)$, la part relative de la vandoise, de l'ablette et du hotu diminue, alors que celle du gardon et du barbeau augmente. C'est l'inverse à fort débit (> $750 \mathrm{~m}^{3} / \mathrm{s}$ ) où la part relative du brochet et du blageon augmente également. Il est légitime de penser que ce résultat reflète la plus ou moins grande capturabilité des espèces en fonction du débit, sans nécessairement signifier que ces espèces modifient leur comportement d'occupation spatiale. Mais l'analyse structurelle des pêches a montré une certaine "anticipation" des modifications liées à la température et aux débits dans les pêches de certains mois. Les deux effets semblent donc exister simultanément, une variation de la capturabilité avec les débits liée à la technique de pêche, et une modification saisonnière de la répartition des poissons sur les rives, le tout indépendamment de la température.

Le groupe chaud $\left(\geq 25^{\circ} \mathrm{C}\right)$ ne concerne que le secteur réchauffé. Par rapport aux analyses structurelles, la station 4 du rejet a été supprimée. La pêche moyenne n'est plus que de 20 individus. Ce groupe se distingue du groupe moyen par l'im- 
portant recul de la vandoise (à peine $10 \%$ des effectifs), une progression importante du goujon et l'absence de truite.

En se référant à la classification biotypologique de Verneaux (1977) qui conjugue les preferenda des espèces et leur nombre, ces trois groupes restent dans l'ordre typologique O-IV représentatif du haut Rhône (types B3 à B9). A côté des espèces rhéophiles, on y rencontre déjà des espèces comme la perche, le gardon, le brochet ou la perche soleil. Le groupe froid, avec seulement 16 espèces présentes ${ }^{(1)}$ et une forte proportion de truite signe une transition B5-B6. Le groupe médian appartient nettement au groupe B6 du fait d'un grand nombre d'espèces (19). Le groupe chaud apparaît plus difficile à classer. II est marqué par le remplacement de la vandoise par le goujon, mais ce sont deux espèces de même type socioécologique d'après cette classification. Cependant, la disparition de la truite, la régression des autres espèces rhéophiles, et la forte proportion d'ablette en font une transition B6-B7, dégradée du fait d'un trop petit nombre d'espèces (17 au lieu de 20-21 d'après la classification). Cette typologie est cependant assez formelle dans la mesure où il ne s'agit pas véritablement de peuplements constitués mais de modifications des associations de poissons en fonction de la température, et qu'elle est faite sur les espèces capturées en bordure.

\subsection{Effet qualitatif du contraste entre veines chaudes et froides}

Parallèlement à l'étude quantitative, l'effet structurel de l'écart thermique entre stations témoins et réchauffées a été recherché. L'analyse est délicate car il faut éviter les interférences liées aux effets thermiques (corrélation entre écart thermique et température), débits et saisons. Pour cela, les stations réchauffées 5 , 6, et 7 (la 4 ne présentant pas suffisamment d'observations) ont été traitées séparément par AFC inter-groupes, en restant en deça du seuil de $25^{\circ} \mathrm{C}$, et en ne conservant que les espèces capturées au moins une fois sur 5 pour limiter le poids des pêches singulières. C'est ainsi que truite et perche soleil ont été supprimées sur les stations 5 et 6 , ainsi que le blageon sur la 5. Parallèlement aux groupes d'écarts thermiques, les autres variables explicatives (températures, débits et mois de l'année) sont projetées pour contrôler leur effet dans la mesure ou là encore, il est impossible de les mélanger équitablement dans tous les groupes. Chaque station est traitée indépendamment, et

(1) On ne retiendra arbitrairement comme espèce présente que les espèces rencontrées au moins une péche sur 20 (5\% des péches), adultes et juvéniles confondus. Le groupe froid passe alors de 25 a 16 especes, le groupe médian de 33 a 19 especes, le groupe chaud de 21 à 17 espèces. 
l'on recherche les points communs entre les analyses. La station $6 G$ a également été traitée en lui affectant les écarts par rapport à la station 6 . Le nombre de pêches par analyse est de 45 (station 5) ou 56-58 (autres stations).

Peu de résultats marquants apparaissent. Seule la perche affiche une affinité pour le même groupe, le groupe de différentiel le plus fort sur toutes les stations. Les autres liaisons traduisent seulement les effets température, débit, ou saison, non totalement supprimés par l'analyse. La station non réchauffée $6 G$ en particulier, ne montre aucune modification de structure en lien avec son écart thermique avec la station 6 .

\section{CONCLUSIONS}

1) Dès que la température dépasse $15^{\circ} \mathrm{C}$, les poissons sont progressivement moins pêchés sur les bordures, notamment les adultes des espèces les plus sténothermes. Aucune espèce ne disparait cependant et la diversité spécifique reste stable. Cette tendance est générale tant sur les stations témoins que réchauffées, mais elle est plus marquée sur ces dernières qui affichent des effectifs globalement plus importants du fait d'une plus grande quantité d'habitat disponible (fig. 4). Du fait de leur plus faible nombre, et surtout de la grande variabilité des effectifs, il n'est pas possible de montrer une différence d'effet thermique entre adultes et juvéniles dont le rapport reste grossièrement de 2/3-1/3 avec notre technique de pêche sur toute la gamme thermique. II semble cependant que l'optimum des juvéniles soit plus large et plus chaud que celui des adultes (fig. 4), mais qu'ils déserteraient plus massivement les bordures au dessus de $29^{\circ} \mathrm{C}$ (fig. $3 \mathrm{C}$ des effectifs et $5 \mathrm{C}$ de la contribution à la diversité).

2) Un seuil quantitatif est observé à $24-25^{\circ} \mathrm{C}$ pour les biomasses (fig. 3a) mais pas pour les effectifs (fig. 3b). Les gros poissons désertent la zone, à l'exception des espèces les plus thermophiles comme la carpe par exemple, espèces par ailleurs assez rares sur ce secteur du Rhône. Au dela de cette température la richesse spécifique commence à décroître (fig. 5b). Ce seuil n'est visible que sur le secteur réchauffé, aucune station témoin n'ayant atteint cette température au cours de la période d'étude.

3) Ce seuil réapparaît sur le plan structurel. A plus de $25^{\circ} \mathrm{C}$, à côté de la trilogie chevaine, ablette et spirlin, la proportion de goujon augmente, mais celle de vandoise, de gardon ou de hotu diminue, et la truite disparaît (fig. 14). On ne peut affirmer que l'effet soit purement thermique dans la mesure où les pêches à plus de $24^{\circ} \mathrm{C}$ sont exclusivement rencontrées sur le secteur réchauffé : il peut s'y mêler un effet lié au contraste thermique entre secteurs réchauffés et froids.

4) Au fur et à mesure que la température continue de croître, adultes et juvéniles quittent la zone réchauffée. La richesse spécifique ne décroît cependant que lentement, seule la 

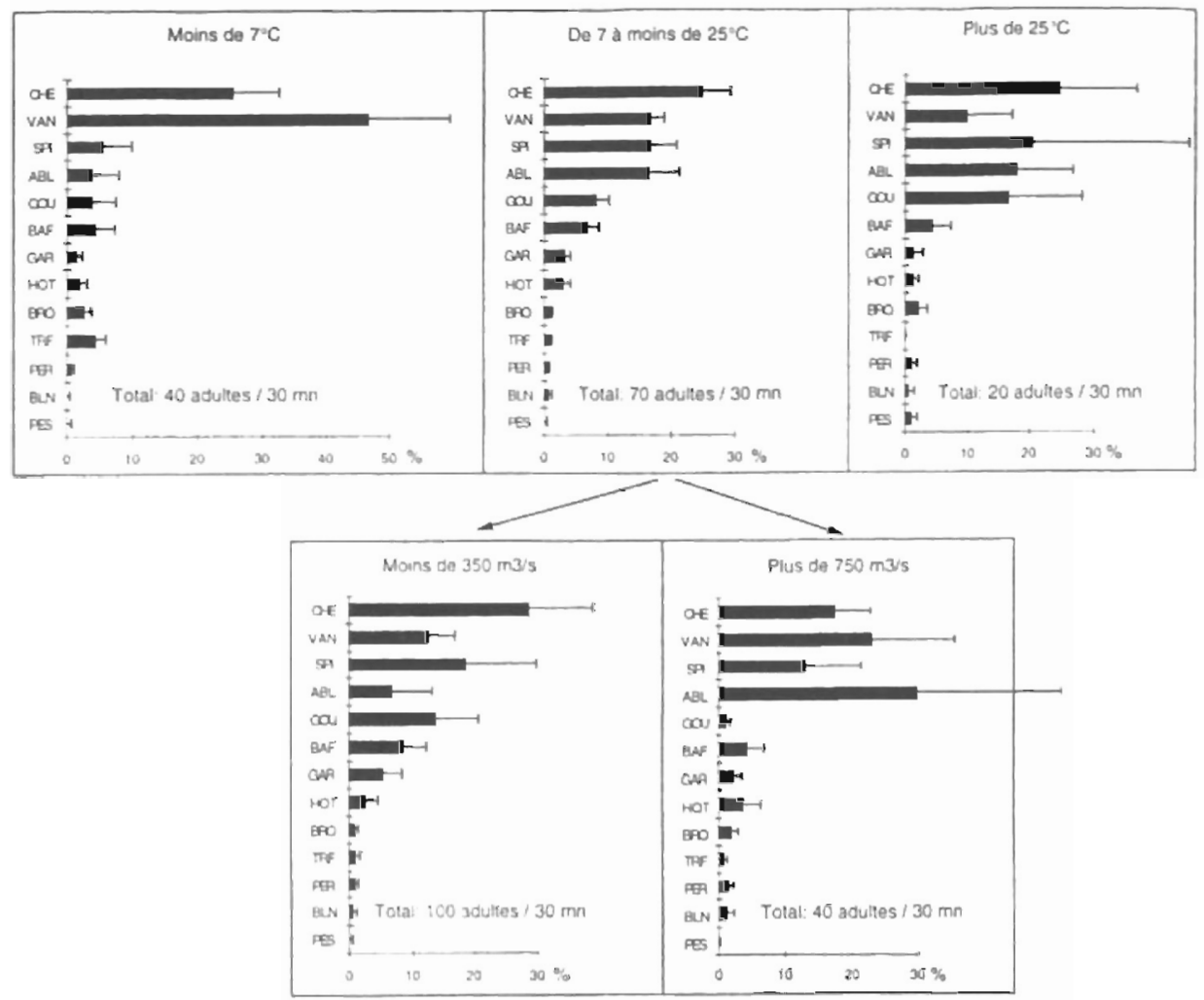

Fig. 14. - Composition spécifique et abondance relative des principaux groupes thermiques et effet du débit. Toutes campagnes et toutes stations sauf stations 8 et 4 .

Fig. 14. - Catches structure of the main thermal groups, and incidence of the discharge, using the data of all campaigns and stations, excepted station 8 and station 4 located in the outflow of the powerplant.

truite et la vandoise disparaissent, la première dès $25^{\circ} \mathrm{C}$, la deuxième vers $27^{\circ} \mathrm{C}$.

5) Un deuxième seuil apparaît à $29^{\circ} \mathrm{C}$ : il ne reste pratiquement plus de poissons sur les bordures réchauffées mis à part quelques ablettes, perches soleil, perches communes, ou chevaines, et ce sont préférentiellement des adultes. La richesse spécifique s'effondre alors.

6) A l'opposé, les pêches à moins de $7{ }^{\circ} \mathrm{C}$ sont marquées par des effec- tifs moins importants et une augmentation relative de la truite et de la vandoise au détriment notamment de l'ablette et du spirlin. Cependant, ces résultats résultent peut-être de la moindre efficacité des pêches électrique en eau froide.

7) Le débit est un élément structurant important, prépondérant dans la gamme thermique centrale (fig. 12). II s'y joint peut-être un facteur saisonnier qui fait que les pêches des mois charnières (mars, juin et août) affi- 
chent déjà des structures proches de la saison qui suit malgré des conditions thermiques et hydrauliques encore typiques de leur saison respective. Ce phénomène explique sans doute en partie la largeur du groupe thermique central $\left(7-24^{\circ} \mathrm{C}\right)$, puisque qu'un même groupe thermique peut inclure des pêches appartenant à des "saisons" différentes du point de vue du poisson. Ces saisons se décomposeraient en une saison hivernale (décembre, janvier, février), une saison printanière à large spectre thermique (mars, avril, mai), une saison estivale également à large spectre thermique (juin, juillet, août), et une saison automnale (septembre, octobre, novembre). La place du mois d'août apparaît incertaine entre le groupe estival et le groupe automnal car bien que thermiquement et hydrauliquement estival la structure de ses pêches semble déjà afficher une tendance automnale.

8) La composition des pêches de juvéniles est extrêmement variable ( $17 \%$ de pêches nulles) et ne peut pas être reliée à un effet thermique, débit ou saison (fig. $9 \mathrm{~b}$ et 10b).

9) L'attraction des veines chaudes semble quantitativement et qualitativement inéxistante en hiver (fig. 8), sauf pour la perche qui montre une affinité systématique pour les forts écarts thermiques. Aucune modification quantitative ou structurelle n'apparaît sur la station non réchauffée $6 G$, en liaison avec le contraste thermique de la station réchauffée 6 située en face. Notons cependant que les données ne sont pas très adap- tées à cette analyse dans la mesure où il serait nécessaire de connaître la durée des épisodes thermiques, et un échantillonnage plus systematique pour espérer mieux dissocier l'effet "écart thermique" des effets "température", "débit", et "saison".

10) Les tendances structurelles observées, qui ne concernent que les adultes, n'expliquent qu'une faible part de la variabilité des pêches. De l'ordre de $10 \%$ pour chacun des effets thermique et hydrologique, et sans doute entre 5 et $10 \%$ pour un effet saisonnier difficile à individualiser, soit au total moins de $30 \%$. Même si une partie des $70 \%$ restants peut être imputée aux modifications interannuelles survenues pendant la période d'étude et non abordées ici, il n'en reste pas moins que la variabilité des pêches est forte, surtout en ce qui concerne les juvéniles, et que la composition d'une pêche donnée reflète d'abord l'aléa de la rencontre avec le poisson et l'aléa de sa capture. II est donc essentiel de garder un effort d'échantillonnage important pour faire ressortir les tendances du peuplement.

11) Ces résultats, qui montrent à la fois une nette différenciation entre stations témoin et réchauffées et une grande homogénéité au sein de ces deux groupes (prépondérance de l'effet thermique sur l'effet "différence morphologiques entre stations"), nous autorisent à les considérer globalement en excluant la station 4 du rejet et la station 8 à la confluence de l'Ain. Le profil moyen des stations réchauffées reste dans la classe B5B6 de Verneaux (1977), malgré la 
quasi-disparition de la truite, la régression de la vandoise et la nette progression du couple ablette-spirlin (fig. 15). Ceci parce que les cyprinidés rhéophiles comme le barbeau et le

hotu restent bien représentés par rapport au groupe témoin. Ce résultat, obtenu pour un écart thermique moyen de $5{ }^{\circ} \mathrm{C}$ avec les stations témoin, est meilleur que les prévisions

\begin{tabular}{|c|c|c|c|c|c|c|c|}
\hline$a$ & & $\begin{array}{l}\text { printemps } \\
\left(604 \mathrm{~m}_{3} / \mathrm{s}\right)\end{array}$ & $\frac{\mathrm{ctc}}{(500 \mathrm{~m} 3 / \mathrm{s})}$ & & $\begin{array}{l}\text { Automne } \\
\left(451 \mathrm{~m}^{3 / 5}\right)\end{array}$ & & $\underset{(130 \mathrm{~m} 3 / \mathrm{s})}{(+70}$ \\
\hline $\begin{array}{l}\text { Stations } \\
\text { témoins }\end{array}$ & 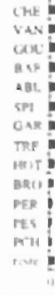 & Sil parhes & 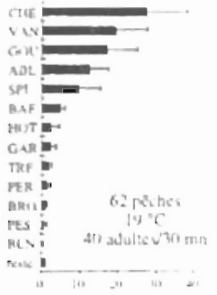 & 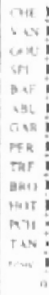 & 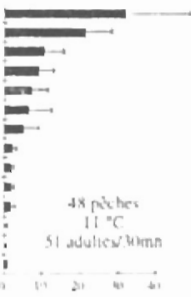 & 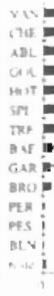 & E. \\
\hline $\begin{array}{l}\text { Shatom } \\
\text { rishaufles }\end{array}$ & 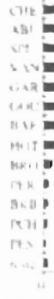 & $=$ & 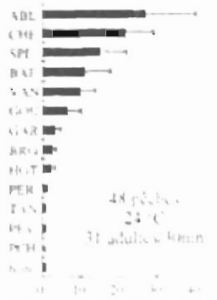 & 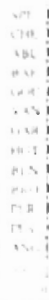 & 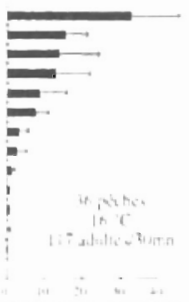 & 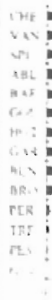 & $=$ \\
\hline
\end{tabular}

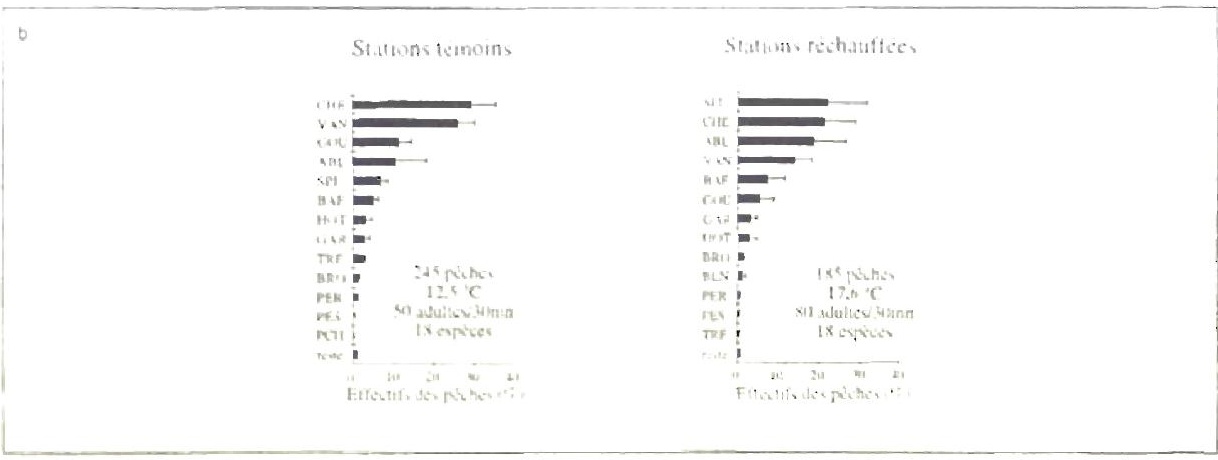

Fig. 15. - Composition spécifique et abondance relative des stations témoins et réchauffées er fonction de la saison (a) ou en moyenne générale (b). Printemps = avril-juin ; été = juillet-septembre automne = octobre-décembre; hiver = janvier-mars. Mention des conditions moyennes (débit, tem pérature, effectif) des pêches. Toutes campagnes ef toutes stations sauf stations 8 et 4 .

Fig. 15. - Comparison of catches structure between heated and control stations versus seasons (a) or mixing all the data (b). Spring : april to june; summer : july to september; automn : octobe to december; winter: janvier to march. Using the data of all campaigns and stations, excepted stations 8 and 4 . 
des études préliminaires qui prévoyaient un glissement typologique d'une unité par tranche de $3^{\circ} \mathrm{C}$ d'élévation de température (Verrel, 1983). Ces prévisions étaient cependant faites sous l'hypothèse d'un mélange complet des masses d'eau et d'une élévation globale de la température. II est très vraisemblable que la juxtaposition des veines froides et chaudes ait contribué à tempérer l'effet du réchauffement, même si aucune différence n'a été décelée dans l'utilisation des contrastes thermiques engendrés. Si l'on y ajoute le fait qu'une veine chaude individualisée se refroidit mieux, il semble que sur ce site, le mauvais mélange des masses d'eau à la sortie de la centrale soit bénéfique.

\section{REMERCIEMENTS}

Les données ont été recueillies dans le cadre de contrats avec Electricité de France, Etudes et Recherches Chatou, pour des synthèses interannuelles et avec l'exploitant du CNPE du Bugey pour les suivis annuels. Un très nombreux personnel de diverses équipes du Cemagref a conduit les pèches, la saisie et l'archivage des données. Qu'il soit collectivement et chaleureusement remercié.

\section{RÉFÉRENCES \\ BIBLIOGRAPHIQUES}

Alabaster J.S., Lloyd R., 1982. Water quality criteria for freshwater fish. Butterworths, 2nd Edition, $361 \mathrm{p}$.

Khalanski M., 1988. Impact hydrobiologique de la centrale du Bugey. Bilan sur la période 1984-1987. Rapport EDFDER HE/31-88.

Magnuson J.J., 1990. Long-term ecological research and the invisible present. Bio Science, 40 (7), 495-501.

Persat $H_{\text {., }}$ 1988. De la biologie des populations de l'ombre commun (Thymallus thymallus, L. 1758) à la dynamique des communautés dans un hydrosystème fluvial aménagé, le Haut-Rhône français. Eléments pour un changement d'échelles. Univ. Lyon I, Thèse de Doctorat d'Etat, 222 p. + figures.

Philippart J.-C., 1979. Observations concernant la pêche à l'électricité dans une rivière à Barbeau. Bulletin Français de Pêche et Pisciculture, 273 (2), 157-172.

Roger M.-Cl., Faessel B., 1989. Effet du réchauffement artificiel de l'eau du Rhône sur le développement et la production de Gammaridae. Hydroécologie Appliquée, 1 (1/2), 53-83.

Roger M.-Cl., Faessel B., Lafont M., 1991. Impact thermique des effluents du centre de production nucléaire du Bugey sur les invertébrés benthiques du Rhône. Hydroécologie Appliquée, $\mathbf{3}$ (1), 63-110.

Thioulouse J., Dolédec S., Chessel D., Olivier J.M., 1995. ADE sofware: multivariate analysis and graphical display of environmental data. In : Guariso G., Rizzoli A. (eds). Software per l'ambiente, Pétron editore, Bologne, pp. 57-62.

Verneaux J., 1977. Biotypologie de l'écosystème eau courante. Détermination approchée de l'appartenance typologique d'un peuplement ichtyologique. C.R. Académie des Sciences de Paris, t. 284, Série D, 675-679.

Verrel J.L., 1983. Impact hydrobiologique des centrales thermoélectriques en cours d'implantation sur le Rhône. Cahiers du laboratoire de Montereau, 14, 45-54. 